\title{
On the entrainment assumption in Schatzmann's integral plume model
}

Article

Accepted Version

Teixeira, M. A. C. and Miranda, P. M. A. (1997) On the entrainment assumption in Schatzmann's integral plume model. Applied Scientific Research, 57. pp. 15-42. ISSN 15731987 doi: https://doi.org/10.1007/BF02528762 Available at https://centaur.reading.ac.uk/29257/

It is advisable to refer to the publisher's version if you intend to cite from the work. See Guidance on citing.

To link to this article DOI: http://dx.doi.org/10.1007/BF02528762

Publisher: Springer Verlag

All outputs in CentAUR are protected by Intellectual Property Rights law, including copyright law. Copyright and IPR is retained by the creators or other copyright holders. Terms and conditions for use of this material are defined in the End User Agreement.

\section{www.reading.ac.uk/centaur}

\section{CentAUR}

Central Archive at the University of Reading

Reading's research outputs online 


\title{
ON THE ENTRAINMENT ASSUMPTION IN SCHATZMANN'S INTEGRAL PLUME MODEL
}

\author{
MigueL A. C. TEIXEIRA and PEDRO M. A. MIRANDA \\ Centro de Geofísica da Universidade de Lisboa Rua da Escola Politécnica, 58, 1250 Lisboa, Portugal \\ January 1997
}

\begin{abstract}
The behaviour of stationary, non-passive plumes can be simulated in a reasonably simple and accurate way by integral models. One of the key requirements of these models, but also one of their less well-founded aspects, is the entrainment assumption, which parameterizes turbulent mixing between the plume and the environment. The entrainment assumption developed by Schatzmann and adjusted to a set of experimental results requires four constants and an ad hoc hypothesis to eliminate undesirable terms. With this assumption, Schatzmann's model exhibits numerical instability for certain cases of plumes with small velocity excesses, due to very fast radius growth. The purpose of this paper is to present an alternative entrainment assumption based on a first-order turbulence closure, which only requires two adjustable constants and seems to solve this problem. The asymptotic behaviour of the new formulation is studied and compared to previous ones. The validation tests presented by Schatzmann are repeated and it is found that the new formulation not only eliminates numerical instability but also predicts more plausible growth rates for jets in co-flowing streams.
\end{abstract}

Key words: Buoyant plume, Entrainment, Integral model, Atmospheric dispersion

\section{Introduction}

Integral models have been used for some time in the simulation of jets and plumes. Early investigations using them are, for example, those of Priestley and Ball (1955), Priestley (1956), Morton et al. (1956) and Morton (1959). More recent survey papers were presented by Briggs (1984) and Weil (1988). Initially, integral models were formulated for vertical plumes in stagnant environments, but generalizations for plumes in crossflows quickly followed, one of the most popular being that of Slawson and Csanady (1967).

Integral models are based on the fundamental laws of Fluid Mechanics: conservation of mass, conservation of momentum, first law of thermodynamics and equation of state. A steady average flow and a plume with a circular cross-section are assumed. Axisymmetric average properties are often prescribed, although values averaged over the plume crosssection or even undefined, self-similar profiles may also be used. After splitting dependent variables into mean and fluctuating components and averaging, the linear perturbation terms vanish. The self-similarity assumption enables one to integrate the equations over the cross-section of the plume and the steady state assumption eliminates all time derivatives. A set of ordinary differential equations is thus obtained, describing the evolution of certain integral quantities along the plume axis.

Integral models were designed to simulate the behaviour of plumes characterized by significant velocity or density perturbations. These perturbations have important consequences to the dynamics, namely through the generation of turbulence. This turbulence is almost always responsible for the largest amount of mixing close to the source. Since the source is generally circular, this self-generated turbulence, as well as the mean property profiles, tend to be initially axisymmetric.

For plumes in cross-flows, there are many factors that contribute to the destruction of axisymmetry: ambient wind, because the transition between the jet and ambient flows can never be axisymmetric except when they have the same direction; buoyancy, because it makes the core of the plume accelerate vertically; ambient stability, because it forces the plume to spread horizontally; and ambient turbulence, because turbulence in the boundary layer is highly anisotropic, causing different horizontal and vertical spread rates. The axisymmetry assumption used in many integral models may only be realistic for plumes 
in stagnant environments or jets in co-flowing, neutrally stratified, streams. Therefore, integral models including a cross-stream can not be expected to describe accurately local properties of the flow.

Slawson and Csanady's model (1967) uses top-hat profiles for plume properties and extends the theory of Morton et al. (1956) to the case of a moving environment. The 3 equations it comprises can be solved analytically.

Many integral models have been developed making use of gaussian profiles. Fox (1970) presented a model for a gaussian plume in a still environment with a parameterization of the entrainment process based on a form of the mechanic energy equation. Hirst (1972) developed a model which included a crossflow, allowed for three-dimensional trajectories and generalized Fox's entrainment hypothesis. Schatzmann (1978) tried to produce a more rigorous model by taking out the Boussinesq approximation and making a careful analysis of the implications of the assumed geometry.

This paper is organized as follows: section 2 presents an outline of Schatzmann's model. In section 3, some previous entrainment assumptions are discussed. Section 4 presents an alternative entrainment assumption based on a standard first-order closure scheme for turbulence. Section 5 includes some validation tests performed using the new assumption. Section 6 contains the main conclusions of this study.

\section{Schatzmann's model}

In the integral model developed by Schatzmann (1978), many of the hypotheses used by Slawson and Csanady (1967) were maintained, since they are necessary to enable integration. However, the entrainment assumption became much more complex, the Boussinesq approximation was not invoked and the profiles used for the plume were gaussian rather than top-hat. In the curvilinear coordinate-system introduced by Hirst (1971) and adopted by Schatzmann (Figure 1), the flow field can be expressed as

$$
\begin{aligned}
& \overline{v_{s}}(s, r)=U_{a} \cos \theta(s)+u^{*}(s) \exp \left(-r^{2} / b(s)^{2}\right) \\
& \overline{v_{r}}(s, r, \varphi)=-U_{a} \sin \theta(s) \sin \varphi+v_{d}(s, r) \\
& \overline{v_{\varphi}}(s, \varphi)=-U_{a} \sin \theta(s) \cos \varphi
\end{aligned}
$$

where $v_{s}$ is the down-axis component of velocity, $v_{r}$ is the radial component, $v_{\varphi}$ is the component tangent to the cross-section boundary and the overbar denotes average values. $U_{a}$ is the ambient wind, assumed constant, $\theta$ is the angle between the plume axis and the horizontal plane, $\varphi$ is the angle measured around the plume axis, $u^{*}$ is the velocity excess at the plume centreline, $v_{d}$ is the radial component of the excess velocity and $b$ is a variable proportional to the plume width. The plume's radial limit $R$ is defined as $R^{2}=2 b^{2}$. Schatzmann's equation set in its final form comprises: mass conservation

$$
\frac{d}{d s}\left[\rho_{a} u^{*} b^{2}+\rho^{*} b^{2}\left(\lambda^{2} U_{a} \cos \theta+\frac{\lambda^{2}}{\lambda^{2}+1} u^{*}\right)\right]+2 b^{2} U_{a} \cos \theta \frac{d \rho_{a}}{d s}=2 \rho_{a} v_{e} R
$$

$s$-momentum budget

$$
\frac{d}{d s}\left[b^{2} u^{* 2}\left(\frac{1}{2} \rho_{a}+\frac{\lambda^{2}}{2 \lambda^{2}+1} \rho^{*}\right)+b^{2} u^{*} U_{a} \cos \theta\left(\rho_{a}+\frac{\lambda^{2}}{\lambda^{2}+1} \rho^{*}\right)\right]=-\lambda^{2} b^{2} \rho^{*} g \sin \theta
$$

thermodynamic equation

$$
\frac{d}{d s}\left[b^{2} \Theta^{*} U_{a} \cos \theta\left(\lambda^{2} \rho_{a}+\frac{\lambda^{2}}{2} \rho^{*}\right)+b^{2} \Theta^{*} u^{*}\left(\frac{\lambda^{2}}{\lambda^{2}+1} \rho_{a}+\frac{\lambda^{2}}{\lambda^{2}+2} \rho^{*}\right)\right]=
$$




$$
=-\left(\frac{d \Theta_{a}}{d s}\right)\left[2 b^{2} U_{a} \cos \theta\left(\rho_{a}+\frac{\lambda^{2}}{2} \rho^{*}\right)+b^{2} u^{*}\left(\rho_{a}+\frac{\lambda^{2}}{\lambda^{2}+1} \rho^{*}\right)\right]
$$

and the $\theta$-equation (momentum budget in a direction perpendicular to $s$ )

$$
\frac{d \theta}{d s}=-\frac{\lambda^{2} b^{2} \rho^{*} g \cos \theta+\rho_{a} U_{a} v_{e} R \sin \theta+\frac{c_{d}}{\pi} \rho_{a} R U_{a}^{2}|\sin \theta| \sin \theta}{b^{2} u^{* 2}\left(\frac{1}{2} \rho_{a}+\frac{\lambda^{2}}{2 \lambda^{2}+1} \rho^{*}\right)+b^{2} u^{*} U_{a} \cos \theta\left(\rho_{a}+\frac{\lambda^{2}}{\lambda^{2}+1} \rho^{*}\right)}
$$

where $\rho_{a}$ is ambient density, $\Theta_{a}$ is ambient potential temperature, $\rho^{*}$ and $\Theta^{*}$ are the density and potential temperature excesses above or below ambient values, $\lambda=1.16$ is the ratio of temperature and momentum plume widths, $g$ is the acceleration of gravity, $c_{d}=2.5$ is a drag coefficient and $v_{e}=-v_{d}(R)$ is the entrainment velocity. An equation formally identical to (4) can be used to describe the mass conservation of a tracer, if $\Theta^{*}$ is replaced by $c^{*}$ and $\Theta_{a}$ by $c_{a}$, where $c_{a}$ is the mass ratio of pollutant in the environment and $c^{*}$ is the corresponding maximum excess. In the drag term of (5), $\sin \theta$ has been replaced by $|\sin \theta|$ to guarantee the appropriate behaviour for both rising and descending plumes. The equation of state presented in Schatzmann and Policastro (1984) completes the set:

$$
\frac{\rho^{*}}{\rho_{a}}=-\frac{\Theta^{*}}{\Theta_{a}+\Theta^{*}}
$$

One advantage of this model over Slawson and Csanady's model is the ability to simulate jets in co-flowing streams. In the former case, this is not possible, since the velocity excess associated to the plume is constrained to be vertical and hence is always perpendicular to the mean wind.

In the present paper, the above equation set was written in dimensionless form and solved numerically using a 4 th order Runge-Kutta procedure.

\section{Some entrainment assumptions}

In integral models, the average mass conservation equation does not have any turbulent terms, since the density-velocity correlations are neglected. The turbulent components of velocity, which appear in linear form, are unable to cause any organized mass displacement, since their averaged value is zero. The increasing average mass flux across the plume section as one moves down-axis, can only be balanced by a non-turbulent (average) lateral mass inflow. This inflow, called entrainment is, nevertheless, associated with turbulence inside the plume. If the flow was entirely laminar, the plume edge would be a material surface and the ambient air would be unable to penetrate it. The radius of the plume only grows because all ambient air that enters it is effectively mixed by turbulence with the previously existing plume air. In this sense, the real entrainment parameterization is not the definition of the mean inflow but the assumption of self-similar profiles, since the maintenance of those profiles implies a very rapid (in fact instantaneous) mixing.

There are two alternative ways of defining the entrainment inflow. One of them is viewing it as an external parameter and simply imposing an entrainment assumption which is dimensionally correct and supported by experimental data. Another possibility is viewing it as an additional variable in the equation set and treating it accordingly. In that case, as there are more variables than equations (for example, Schatzmann's set, as presented above, contains 5 equations and 6 unknowns: $\theta, b, u^{*}, \Theta^{*}, \rho^{*}$ and $v_{e}$ ), a new equation is required. The mechanic energy equation, which is obtained by multiplying the momentum equation by velocity, is often used to close the set. Naturally, this equation contains a new unknown: $\overline{v_{s}^{\prime} v_{r}^{\prime}}$, the correlation of turbulent perturbations of the radial and down-axis components of velocity, which has to be related to the mean variables of the model if a unique solution is to be found. 
Many entrainment assumptions have been developed. Using the present notation, Morton et al. (1956), who treated the case of vertical plumes in stagnant environments, simply stated that

$$
\varepsilon=\alpha
$$

where $\varepsilon$ is the dimensionless entrainment coefficient $\varepsilon=R v_{\epsilon} / b u^{*}$ and $\alpha$ is a dimensionless constant.

Fox (1970) used the mechanic energy equation and the similarity assumption

$$
\overline{v_{s}^{\prime} v_{r}^{\prime}}=u^{* 2}(s) h(r / b)
$$

where $h$ is an unknown function of $r / b$ to obtain the result

$$
\varepsilon=\alpha_{1}+{\frac{\alpha_{2}}{\mathcal{F}}}^{2}
$$

where $\alpha_{1}$ and $\alpha_{2}$ are dimensionless constants depending on the form of function $h$ and where $\mathcal{F}$ is the densimetric Froude number, defined as

$$
\mathcal{F}^{\epsilon}=\frac{\Gamma^{* \epsilon}}{\}\left\lfloor\rho^{*} / \rho_{\dashv}\right.}
$$

Hirst (1971) followed the steps of Fox and generalized his entrainment assumption to the case of plumes with non-vertical paths:

$$
\varepsilon=\alpha_{1}+\frac{\alpha_{2} \sin \theta^{2}}{\mathcal{F}}
$$

The same author (Hirst, 1972) subsequently developed a model of three-dimensional plume in a crossflow, and further generalized the above equation, but instead of using the same kind of similarity assumptions, added some empirical terms based on the work of Hoult et al.(1969). In the present notation, and for the particular case of a plume with a 2dimensional trajectory, the resulting entrainment assumption takes the form

$$
\varepsilon=\left(\alpha_{1}+\frac{\alpha_{2} \sin \theta^{2}}{\mathcal{F}}\right)\left(\left|1-\frac{U_{a}}{u^{*}} \cos \theta\right|+\alpha_{3} \frac{U_{a}}{u^{*}} \sin \theta\right)
$$

Schatzmann (1979) introduced a generalized form of Fox's mechanic energy equation, taking into account a cross-flow, which for the Boussinesq approximation reads

$$
\frac{d}{d s}\left[b^{2} u^{* 2}\left(u^{*}+\frac{3}{2} U_{a} \cos \theta\right)\right]=-\frac{6}{\lambda^{2}+1} b u^{* 3} \frac{\lambda^{2} \sin \theta^{2}}{\mathcal{F}}-24 \frac{u^{*}}{b^{2}} \int_{0}^{\infty} \overline{v_{s}^{\prime} v_{r}^{\prime}} \exp \left(-r^{2} / b^{2}\right) r^{2} d r
$$

and eliminated the turbulent correlation inside the integral by using the $s$-momentum equation integrated indefinitely with respect to $r$. His calculations produced an expression of the form

$$
\varepsilon=\frac{\frac{d b}{d s}+2 A_{2} \frac{\sin \theta^{2}}{\mathcal{F}}+\left(\frac{3}{2} \frac{d b}{d s}+A_{5} b \sin \theta \frac{d \theta}{d s}\right) \frac{U_{a}}{u^{*}}}{2+A_{3} \frac{U_{a}}{u^{*}} \cos \theta}
$$

where the $A_{i}$ are constants. But he neglected the terms between brackets in the numerator, and replaced the first term by a constant, with a value adjusted to the particular case of a momentum jet in a quiescent atmosphere. He also multiplied this expression by a factor, to account for the positive contribution to entrainment from the shear perpendicular to 
the plume axis, associated with the vortex pair existing in the real flow field (Moussa et al., 1977). The final expression obtained was

$$
\varepsilon=\frac{2 A_{1}+2 A_{2} \frac{\sin \theta^{2}}{\mathcal{F}}}{2+A_{3} \frac{U_{a}}{u^{*}} \cos \theta}\left(1+A_{4} \frac{U_{a}}{u^{*}}|\sin \theta|\right)
$$

where $A_{1}=0.057, A_{2}=-0.67, A_{3}=10$ and $A_{4}=2$ are dimensionless constants.

It is easy to show that an equation relatively similar to (14) can be found with much less effort, by manipulating equations (2) and (3), with the Boussinesq approximation

$$
\varepsilon=\frac{\frac{d b}{d s}-\frac{\lambda^{2} \sin \theta}{\mathcal{F}}{ }^{2}+b \sin \theta \frac{d \theta}{d s} \frac{U_{a}}{u^{*}}}{2+2 \frac{U_{a}}{u^{*}} \cos \theta}
$$

This expression bears a remarkable resemblance to (14), but the constants have been replaced by particular values. Neglecting the last term in the numerator, as suggested by Schatzmann, the only difference between (14) and (16) is the value of $A_{3}$, which is 10 in the first case and 2 in the second. However, for the experiment trajectory shown in Figure 2 , it can be seen that it is completely unacceptable to use $A_{3}=2$ and to keep the other constants unchanged. In that figure, $r_{1 / 2}$ is the radius where the velocity excess reaches half of its maximum value and $D$ is the source diameter. The conditions for run $4-1$, which will be analyzed in more detail in a later section, can be found in table I.

Replacing the derivatives in a general definition such as (14) by values found for particular cases is certainly not a very satisfactory procedure and seems contradictory to the general approach used. That choice seems to arise because the use of the $s$-momentum equation to eliminate the turbulent correlation in (13), without the introduction of any new hypothesis, does not allow for elimination of all the derivatives in (14). The procedure followed by Fox (1970) and Hirst (1971) appears to be theoretically much more satisfactory, because the similarity assumption used by them can be interpreted as an independent constraint, and the constants $\alpha_{1}$ and $\alpha_{2}$ are obtained in a consistent manner as a consequence of this constraint. In the following section, a slightly more specific choice will be proposed.

\section{An alternative entrainment assumption}

One possible way of parameterizing the turbulent correlation in the mechanic energy equation is using a simple first-order turbulence closure scheme. Assuming, consistently with Schatzmann's model, that the turbulent fluxes are essentially radial, it follows that

$$
\overline{v_{s}^{\prime} v_{r}^{\prime}}=-K(s) \frac{\partial \overline{v_{s}}}{\partial r}
$$

where $K$ is a turbulent diffusivity that may depend on coordinate $s$. The mechanic energy equation that will be used must be consistent with Schatzmann's set, which generally does not assume the Boussinesq approximation. The generalization of (13) to that case is

$$
\begin{aligned}
& \frac{d}{d s}\left[\rho_{a} b^{2} u^{* 2}\left(u^{*}+\frac{3}{2} U_{a} \cos \theta\right)+3 \rho^{*} b^{2} u^{* 2}\left(\frac{\lambda^{2}}{3 \lambda^{2}+1} u^{*}+\frac{\lambda^{2}}{2 \lambda^{2}+1} U_{a} \cos \theta\right)\right]= \\
= & -\frac{6}{\lambda^{2}+1} \rho_{a} b u^{* 3} \frac{\lambda^{2} \sin \theta^{2}}{\mathcal{F}}-24 \frac{u^{*}}{b^{2}} \int_{0}^{\infty} \overline{v_{s}^{\prime} v_{r}^{\prime}}\left[\rho_{a}+\rho^{*} \exp \left(-r^{2} / \lambda^{2} b^{2}\right)\right] \exp \left(-r^{2} / b^{2}\right) r^{2} d r
\end{aligned}
$$


Inserting (17) into (18), the last term containing the turbulent correlation becomes

$$
-6 K u^{* 2}\left(\rho_{a}+\frac{4 \lambda^{4}}{\left(2 \lambda^{2}+1\right)^{2}} \rho^{*}\right)
$$

and, provided that a suitable definition is found for $K$, the equation set, (2)-(3)-(4)-(5)(6)-(18) can be solved without problems. When the idea is simply to solve the equation set numerically, it is unnecessary to derive an explicit expression for $\varepsilon$. In fact, that expression is too complex for the general case and will not be presented here. However, before testing the new entrainment assumption numerically, it is instructive to compare the behaviour of Schatzmann's set subject to the entrainment assumptions of Schatzmann (hereafter referred to as SE) and to the one presented now (hereafter referred to as FOC), for some simple asymptotic situations. For that purpose, if is useful to obtain $\varepsilon$ explicitly. Fortunately, in many simple situations, the Boussinesq approximation is acceptable. With that approximation, the equation set is simplified and $\varepsilon$ is found to be

$$
\begin{aligned}
& \varepsilon=\frac{-\left[\left(\lambda^{2}-\frac{1}{2}+\left(4 \lambda^{2}+1\right) \frac{U_{a}}{u^{*}} \cos \theta\right) \frac{1}{\lambda^{2}+1}+3 \frac{U_{a}^{2}}{u^{* 2}} \cos ^{2} \theta\right] \frac{\lambda^{2} \sin \theta^{2}}{\mathcal{F}}}{\left(1+\frac{U_{a}}{u^{*}} \cos \theta\right)\left(1+3 \frac{U_{a}}{u^{*}} \cos \theta\right)\left(\frac{1}{2}+\frac{U_{a}}{u^{*}} \cos \theta\right)+\left(\frac{5}{4}+\frac{3}{2} \frac{U_{a}}{u^{*}} \cos \theta\right) \frac{U_{a}^{2}}{u^{* 2}} \sin ^{2} \theta} \\
& +\frac{-\left(\frac{5}{4}+\frac{3}{2} \frac{U_{a}}{u^{*}} \cos \theta\right) \frac{c_{d}}{\pi} \sqrt{2} \frac{U_{a}^{3}}{u^{* 3}} \sin ^{2} \theta|\sin \theta|+\frac{3 K}{b u^{*}}\left(\frac{1}{2}+\frac{U_{a}}{u^{*}} \cos \theta\right)}{\left(1+\frac{U_{a}}{u^{*}} \cos \theta\right)\left(1+3 \frac{U_{a}}{u^{*}} \cos \theta\right)\left(\frac{1}{2}+\frac{U_{a}}{u^{*}} \cos \theta\right)+\left(\frac{5}{4}+\frac{3}{2} \frac{U_{a}}{u^{*}} \cos \theta\right) \frac{U_{a}^{2}}{u^{* 2}} \sin ^{2} \theta}
\end{aligned}
$$

On the other hand, the expression relating the radius growth rate $d b / d s$ to $\varepsilon$ is also relatively simple

$$
\begin{gathered}
\frac{d b}{d s}=\frac{\varepsilon\left[2\left(\frac{1}{2}+\frac{U_{a}}{u^{*}} \cos \theta\right)\left(1+\frac{U_{a}}{u^{*}} \cos \theta\right)+\frac{U_{a}^{2}}{u^{* 2}} \sin ^{2} \theta\right]}{\frac{1}{2}+\frac{U_{a}}{u^{*}} \cos \theta} \\
+\frac{\frac{c_{d}}{\pi} \sqrt{2} \frac{U_{a}^{3}}{u^{* 3}} \sin ^{2} \theta|\sin \theta|+\frac{\lambda^{2} \sin \theta^{2}}{\mathcal{F}}\left(\frac{1}{2}+2 \frac{U_{a}}{u^{*}} \cos \theta\right)}{\frac{1}{2}+\frac{U_{a}}{u^{*}} \cos \theta}
\end{gathered}
$$

enabling an easy analytical treatment.

\subsection{Plume in a still environment}

Consider a momentum jet in a neutral atmosphere without wind. In that case, Schatzmann's set consists of only two equations: mass conservation

$$
\frac{d}{d s}\left(b^{2} u^{*}\right)=2 R v_{e}
$$

and $s$-momentum budget

$$
\frac{d}{d s}\left(b^{2} u^{* 2}\right)=0
$$

For this particular situation, (15) is equivalent to

$$
\varepsilon=A_{1} \Rightarrow v_{e}=\left(A_{1} / \sqrt{2}\right) u^{*}
$$

and (20) reduces to

$$
\varepsilon=\frac{3 K}{b u^{*}} \Rightarrow v_{e}=\frac{3 K}{R}
$$


(21) can be used to find expressions for the radius growth rate

$$
\begin{aligned}
& \frac{d b}{d s}=2 A_{1} \\
& \frac{d b}{d s}=\frac{6 K}{b u^{*}}
\end{aligned}
$$

respectively using SE and FOC. It is known since long ago (Morton et al., 1956) that measurements indicate a constant $d b / d s$ for vertical momentum jets. Such result is reproduced by SE, and may also be reproduced by FOC as long as it is assumed

$$
K=\beta b u^{*}
$$

where $\beta$ is a dimensionless constant. This, in turn, leads to

$$
\varepsilon=3 \beta \Rightarrow v_{e}=(3 \beta / \sqrt{2}) u^{*}
$$

which is similar to (24) and reproduces the results of Morton et al. (1956).

An expression for the diffusivity like (28) was suggested by Slawson and Csanady (1967) for the initial phase of plume dispersion, when self-generated turbulence is dominant. This is clearly the most obvious choice suggested by dimensional analysis. For a momentum jet in a quiescent atmosphere, $b u^{*}$ is a constant for each plume under consideration, as can be seen from (23), although it varies according to the initial conditions. More sophisticated definitions of $K$ may be searched for, making use of experimental measurements, with a larger number of parameters being taken into account. It is, in principle, possible to obtain $K$, implicitly or explicitly, as a function of coordinate $s$, in the same way as in classical dispersion theory. As a first approach, (28) may be generalized to the case of buoyant plumes in cross-flows.

Having in mind that $b^{2}=2 \sigma^{2}$, where $\sigma$ is the standard deviation of the velocity profile, (27) can be expressed in the form

$$
u^{*} \frac{d \sigma^{2}}{d s}=6 K
$$

which relates the evolution of variance to the diffusion coefficient, as in passive plume dispersion (Csanady, 1973). The term on the left is the material derivative of variance in the middle of the plume and $K$ is a diffusivity associated with self-generated turbulence.

If the zero-buoyancy hypothesis is abandoned but all other assumptions remain, (15) becomes

$$
\varepsilon=A_{1}+{\frac{A_{2}}{\mathcal{F}}}^{2} \sin \theta
$$

which is Hirst's entrainment assumption and (20) turns into

$$
\varepsilon=3 \beta-\frac{2 \lambda^{2}-1}{\lambda^{2}+1} \frac{\lambda^{2}}{\mathcal{F}} \sin \theta
$$

which has the same form. Fox's entrainment assumption would be found if the plume trajectory was further constrained to be vertical $(\sin \theta=1)$. The equivalence between SE, FOC and Fox's formulation is not complete because Schatzmann uses an empirical value for $A_{2}$.

The main conclusion to take from the above analysis is that, in a still environment, SE and FOC are formally equivalent and are both consistent with previous work.

\subsection{Momentum Jet in a CO-FLowing StREam}

The second asymptotic situation to be considered is that of a momentum jet in a co-flowing stream with a relatively small velocity excess $\left(u^{*} \ll U_{a}\right)$. The relevant equations are the 
same as in the previous subsection: conservation of mass and momentum, but the latter has to be slightly changed to:

$$
\frac{d}{d s}\left[b^{2} u^{*}\left(U_{a}+\frac{1}{2} u^{*}\right)\right]=0
$$

With these conditions, SE and FOC take, respectively, the forms

$$
\begin{gathered}
\varepsilon=2 \frac{A_{1}}{A_{3}} \frac{u^{*}}{U_{a}} \Rightarrow v_{e}=\frac{2 A_{1}}{\sqrt{2} A_{3}} \frac{u^{* 2}}{U_{a}} \\
\varepsilon=\frac{K}{b} \frac{u^{*}}{U_{a}^{2}} \Rightarrow v_{e}=\frac{K}{R} \frac{u^{* 2}}{U_{a}^{2}}
\end{gathered}
$$

and, again, the radius growth rate can be determined for each case, yielding

$$
\begin{aligned}
& \frac{d b}{d s}=4 \frac{A_{1}}{A_{3}} \\
& \frac{d b}{d s}=\frac{2 K}{b U_{a}}
\end{aligned}
$$

respectively for SE and FOC. If the diffusivity definition (28) is accepted to hold, then (37) can be expressed as

$$
\frac{d b}{d s}=2 \beta \frac{u^{*}}{U_{a}}
$$

which can also be written

$$
U_{a} \frac{d \sigma^{2}}{d s}=2 K
$$

again, reproducing the known result from classical dispersion theory.

Using (33) and (38), it can readily be concluded that, with FOC,

$$
\frac{d b}{d s}=\frac{\text { const }}{b^{2}} \Rightarrow b=(\text { const } \times s+\text { const })^{1 / 3}
$$

and it becomes clear that the two approaches are qualitatively different. When $u^{*} \rightarrow 0$, SE predicts $d b / d s \rightarrow$ const., while FOC leads to $d b / d s \rightarrow 0$. This last result seems the most logical in a model where only self-generated turbulence is taken into account, since any plume growth happening when $u^{*}=0$ and $U_{a} \neq 0$ could only be attributed to external turbulence. Besides, the second result is supported by dimensional analysis.

\subsection{Evaluation of numerical constants}

In the first asymptotic situation, the performance of the two entrainment assumptions was found to be similar. Then, if (26) and (27) are to be perfectly equivalent, one must have:

$$
A_{1}=3 \beta
$$

which implies $\beta=0.019$, a value to be used in future calculations. On the other hand, for (31) and (32) to be compatible, it would be necessary that

$$
A_{2}=-\frac{2 \lambda^{2}-1}{\lambda^{2}+1} \lambda^{2}
$$

This equality is not exactly verified for SE, since the right hand-side takes the value 0.97 , using $\lambda=1.16$ and $A_{2}=-0,67$ in (15), but the sign and the order of magnitude are correct. A remarkable aspect about this equation is that it relates a constant in the 
entrainment assumption to the ratio of heat and momentum turbulent diffusivities. This result is by no means new and was obtained, for example, by Hirst (1971).

It may be further referred that the proposed FOC, together with definition (28) of the diffusion coefficient, is a particular case of Fox's similarity approach. In fact, (17) is equivalent to (8) if the similarity function $h$ is defined as

$$
h(r / b)=2 \beta \frac{r}{b} \exp \left(-\frac{r^{2}}{b^{2}}\right)
$$

\subsection{Plume in a CRoss-Flow}

The situations considered up to now do not take into account a crossflow, because its existence keeps definition (20) in a much more complex form and an analytical treatment is not feasible. However, numerical tests using the new turbulence closure show that, while predictions for vertical plumes are very similar to Schatzmann's and agree well with experimental data, plumes in cross-flows tend to rise too much (Figure 3 ). This is undoubtedly due to insufficient entrainment caused by not taking into consideration the effects on mixing of the shear associated to the vortex pair in the plume flow. One possible way of parameterizing this effect would be by including in (20) a factor like the one used in (15). But this procedure would be against the mathematical rigour of the latter equation, which must be consistent with the other equations of the set. A more adequate choice seems to be the introduction of a similar factor in the diffusivity $K$, which is only constrained to be a function of coordinate $s$, that is

$$
K=\beta b u^{*}\left(1+\gamma \frac{U_{a}}{u^{*}}|\sin \theta|\right)
$$

$\gamma$ is a new dimensionless constant, which the calibration against experimental data showed to be about 4.5. It is worth noting that this modification does not change any of the previous asymptotic results, since in one of the situations considered $U_{a}=0$ and in the other $\sin \theta=0$.

Other, more complicated definitions, may be found, that fit experimental data more effectively. The present one has the advantage of only requiring the adjustment of two constants $(\beta$ and $\gamma)$.

As a preliminary numerical test, it would be interesting to investigate if the present model reproduces the well-known $2 / 3$ and $1 / 3$ power laws, respectively for the rise of buoyant plumes and momentum jets released into neutrally stratified cross-flows and if the new entrainment assumption improves the agreement with these laws in any way. Results intended to clarify this point can be seen in Figure 4, for simple initial conditions. The trajectories have been represented in graphs with logarithmic axes to facilitate the analysis. Although these results refer to rather particular situations, the overall qualitative behaviour was seen to be roughly the same if the conditions were altered.

The main aspects that can be noticed are the apparent agreement with some kind of power law, in the case of the buoyant plume, specially after $y / D>50$, where $D$ is the source diameter, and the agreement with a power law only for a few initial diameters, in the case of a momentum jet. The slope of the straight portions of the curves was evaluated. For buoyant plumes, a linear fit was performed for $y / D>70$ and the slope of the straight line was found to be 0.68 for FOC and 0.683 for SE. This corresponds to a deviation of about $2 \%$ from the expected $2 / 3$ slope, in both cases. For the momentum jet, the linear fit was performed for $y / D<5$ and the slopes are, in the same order, 0.443 and 0.437 respectively, corresponding to a deviation of about $25 \%$ from $1 / 3$. Figure 4 shows that, for a momentum jet, the slope of the curve decreases for bigger distances from the 
source, thereby approximating the expected slope, but the curve does not become straight, indicating a dependence of a different kind from a power law.

The $2 / 3$ power law is thus approximately respected by buoyant plumes but the same does not apply to the $1 / 3$ power law for momentum jets. The differences in the behaviour of the model subject to the two entrainment formulations may be considered insignificant for this purpose and it may be concluded that no improvement is achieved using the FOC.

\section{Numerical experiments}

In this section, Schatzmann's validation tests are repeated. These are numerical tests performed without the Boussinesq approximation, where the equation set is solved using a 4th-order Runge-Kutta method. In table I, the relevant ambient and initial conditions are described for each run. All experimental data have been taken from Schatzmann (1979). The last test included in Schatzmann's paper was not performed, since the present model does not allow for a vertically varying ambient stratification. It would be relatively straightforward to include this capability in the model, but it would not add much to the conclusions. The initial Froude number is defined as

$$
\mathcal{F} \nabla=\frac{\Pi_{\mid}^{*}}{\sqrt{\jmath \mathcal{D}}}
$$

The initial conditions shown are conditions at the source, but the model may only be applied after the flow establishment zone. The expressions used to relate parameters at the source and at the end of the flow establishment zone can be found, for example, in Davidson (1986).

In runs $1-1$ to $2-9$, results obtained with the FOC are exactly equal to those obtained with SE, so the corresponding graphs will not be presented. The results of runs 2-10 to 3-7, displayed in Figures 5 and 6, also differ very little between the two entrainment formulations, although the FOC agrees slightly better with experimental data in some cases, predicting a slightly faster radius growth. Runs 4-1, 4-2 and 4-3, presented in Figure 7 , correspond to a situation where there is an important qualitative difference between the results obtained with the SE and the FOC. As it was expected from (40), the FOC predicts a radius growth that is slower, and a centreline velocity decay that is also consistently slower. The radius growth is somewhat more in agreement with the data, but the same can not be said about the centreline velocity excess, which in Run 4-3, is more than 4 times the experimental values, far from the source.

In runs 5-1 to 6-7, presented in Figures 8 and 9, the FOC shows in general a slightly better agreement with experimental data than SE. The same happens in runs 7-14 and 715, of Figure 10, but not in runs 7-17 and 7-18, where the agreement of the FOC is a little worse. The experimental data of the last 4 runs come from real stack plume measurements and it appears in some of them that there is influence from ambient large scale turbulence (see for example run 7-14). Apparently the averaging process that should have been carried out for these data to be comparable with the model output was inadequate. Therefore, agreement of theory with data in those cases has a considerable component of luck. The following sections will discuss some of the results in more detail.

\subsection{JET in A CO-FLOWING STREAM}

Although individually the radius and velocity excess behave differently for SE and FOC, these two variables are closely related. While using SE the velocity excess predictions are good but the radius growth is excessive, using the FOC the radius growth is acceptable but the velocity excess is overpredicted, as can be seen in Figure 7 . That behaviour may 
be explained making use of the s-momentum equation, which can be expressed as

$$
b^{2} u^{*}\left(U_{a}+\frac{1}{2} u^{*}\right)=\text { const. }
$$

For a given $U_{a}$ and given initial conditions, (46) defines a relation between $b$ and $u^{*}$ that can not be escaped. It can thus be concluded that a good prediction for both variables is not possible in the framework of this integral model, using the available data. It would be interesting to understand the reasons of such limitation. For that purpose a more detailed discussion of the model is required.

The assumptions of axisymmetry and gaussian profiles seem to be acceptable for a plume in a co-flowing stream. So, one possible explanation for the differences between theory and data is the neglect of certain terms like those concerning the ambient turbulence and the longitudinal turbulent fluxes. Including all the turbulent terms in the momentum equation leads to:

$$
\frac{d}{d s}\left[b^{2} u^{*}\left(U_{a}+\frac{1}{2} u^{*}\right)\right]=-2 R \overline{v_{s}^{\prime} v_{r}^{\prime}}(R)-2 \int_{0}^{\infty} \frac{\partial \overline{v_{s}^{\prime 2}}}{\partial s} r d r
$$

The left-hand side and the last term on the right-hand side of this equation do not depend on $R / b$, because the integrals used in their calculation converge as $R / b \rightarrow \infty$ and these terms are approximated accurately as long as this quantity is sufficiently large. To use for example, $R / b=10$ or $R / b=\infty$ produces almost exactly the same result. But the first term on the right-hand side depends on $R$ and thus would be different for each ratio $R / b$ chosen, for a given $\overline{v_{s}^{\prime} v_{r}^{\prime}}$ distribution. So, this term has to be zero. The physical interpretation of this constraint is that the assumption of a shear-free environment ( $U_{a}=$ const.) used in the calculation of the left-hand side is inconsistent with a non-zero turbulent correlation in the environment. In the case of a wind with considerable shear, the left-hand side of (47) would not be valid, since it was derived taking $U_{a}$ as a constant and a simple mixinglength reasoning shows that this implies $\overline{v_{s}^{\prime} v_{r}^{\prime}}=0$. To treat the shear-wind case, a whole new equation set would be required. Even so, it must be noted that (47) would have limited applicability since it was derived taking the correlation as axisymmetric, and that kind of symmetry is very unlikely for ambient turbulence.

The last term on the right-hand side concerns only turbulence inside the plume, because ambient turbulence is assumed not to change along $s$. Using dimensional reasoning, that term may be parametrized as

$$
-c \frac{d}{d s}\left(b^{2} u^{* 2}\right)
$$

where $c>0$ is a constant. Inserting (48) into (47) yields

$$
b^{2} u^{*}\left[U_{a}+\left(\frac{1}{2}+c\right) u^{*}\right]=\text { const. }
$$

But it is easily shown that this even worsens the agreement with experimental data, since, for the same velocity excess, (49) predicts a faster radius growth than (47).

Therefore, the discrepancy between (49) and experimental results may be attributed to experimental errors, because this is a situation where Schatzmann's geometry assumptions are almost exactly satisfied.

Schatzmann (1979) points out that the experimental data of Fink (1974) indicate a growth of the type

$$
\frac{d b}{d s}=\frac{\text { const }}{b}
$$


for jets in co-flowing streams. It can be shown using (33) that this implies, for relatively small $u^{*}$,

$$
\frac{d b}{d s}=\text { const } \times \sqrt{u^{*}}
$$

or

$$
K \propto \sqrt{u^{*}}
$$

Although a turbulent diffusivity with this asymptotic behaviour would give results somewhere in between those of Schatzmann and those obtained with the FOC, it would not solve the main problem, which is the inability to match simultaneously $b$ and $u^{*}$, because these variables are related by the momentum equation and that equation does not depend on the diffusion coefficient.

\subsection{Plume in a CRoss-flow With a SMall Velocity excess}

A careful examination of the results displayed in Figure 10 shows that, in some cases, the solution of the integral model using SE fails to converge before the end of the run. This happens because at a certain stage the radius begins to grow very fast and the numerical resolution scheme is forced to adopt increasingly smaller integration steps. Schatzmann (1979) pointed out that his program tended to be unstable when $U_{a} / u_{j}^{*}>0.7$, which may be a sign of this instability. It can be proved very simply that this behaviour is inherent to the analytical form of Schatzmann's entrainment assumption.

Consider a case where $u^{*}$ tends to zero but where the plume is not aligned with the ambient wind (e.g. a buoyant plume in an unstable atmosphere). Then, (15) takes the form

$$
\varepsilon=\left(2 A_{1}+2 A_{2} \frac{\sin \theta^{2}}{\mathcal{F}}\right) \frac{A_{4}}{A_{3}}|\tan \theta|
$$

and (21) reduces asymptotically to

$$
\frac{d b}{d s}=\left(2 \frac{U_{a}}{u^{*}} \cos \theta+\frac{U_{a}}{u^{*}} \sin \theta \tan \theta\right) \varepsilon+2 \lambda^{2} \sin \theta \frac{g b \rho^{*} / \rho_{a}}{u^{* 2}}+\frac{c_{d}}{\pi} \sqrt{2} \frac{U_{a}^{2}}{u^{* 2}} \sin \theta|\sin \theta| \tan \theta
$$

For a sufficiently small $u^{*}$,using (53), it is obvious that all terms in (54) diverge, imposing a radius growth that tends to infinite. This causes the plume to spread too quickly when it does not approach a horizontal trajectory sufficiently fast, particularly for big initial ratios $U_{a} / u^{*}$. Such problem does not arise with the new entrainment assumption, since (53) is replaced by

$$
\varepsilon=\frac{-2 \lambda^{2} \sin \theta \frac{g b \rho^{*} / \rho_{a}}{u^{* 2}}-\frac{c_{d}}{\pi} \sqrt{2} \frac{U_{a}^{2}}{u^{* 2}} \sin \theta|\sin \theta| \tan \theta+\frac{2 K}{b u^{*}} \frac{u^{*}}{U_{a}} \frac{1}{\cos \theta}}{2 \frac{U_{a}}{u^{*}} \cos \theta+\frac{U_{a}}{u^{*}} \sin \theta \tan \theta}
$$

and hence (54) simply reduces to

$$
\frac{d b}{d s}=\frac{2 K}{b u^{*}} \frac{u^{*}}{U_{a}} \frac{1}{\cos \theta}
$$

forcing a type of growth which is never faster than linear, even when the factor related to the vortex pair is taken into account in the definition of $K$. The fact that the buoyancy and drag terms cancel is surely a consequence of the internal consistency of the FOC, where the radius growth is essentially related to the diffusivity $K$. In fact, (56) can be expressed as

$$
U_{a} \cos \theta \frac{d \sigma^{2}}{d s}=2 K
$$


which is a generalization of (39) to weak plumes in cross-flows. A comparison of SE and FOC for an ambient wind equal to the initial velocity excess is displayed in Figure 11. For bigger ratios $U_{a} / u_{j}^{*}$, the radius explodes even closer to the source, using SE, but nothing similar happens when the FOC is used instead. It must be stressed that it is important to use the same kind of approximation in both (2)-(3)-(4)-(5)-(6) and (18) for these results to hold. If, for example, the main equation set does not assume the Boussinesq approximation but the kinetic energy equation assumes that approximation, the abovementioned cancellation does not happen exactly and numerical problems may persist. That is why, in this work, the general form (18), not using the Boussinesq approximation, had to be derived.

\section{Conclusions}

A first-order turbulence closure (FOC) has been applied to Schatzmann's integral model in order to obtain a new entrainment assumption. With the form suggested by Slawson and Csanady (1967) for the turbulent diffusivity, the FOC was found to be a particular case of the similarity approach of Fox (1970) and Hirst (1971). Is is, however, applicable to more general situations, because in its derivation a crossflow was considered and the Boussinesq approximation was not assumed. In the general case, entrainment is found by solving the equation set including the mechanic energy equation. For the Boussinesq approximation, an explicit entrainment formula was derived and studied for some particular cases. It was concluded that this formula reduced to Hirst's entrainment formula for a plume in a still environment, to Fox's for a vertical plume in a still environment, and to Morton et al.'s for a momentum jet in a still environment. For a jet in a co-flowing stream, some important qualitative differences between SE and the FOC have been found, with the FOC predicting a slower radius growth. The relation between the radius and the velocity excess was found to depart from measurements for both approaches, and to be constrained by the momentum equation, where the entrainment assumption is not needed. For plumes in cross-flows, it could be concluded that an additional factor has to be introduced in order to simulate the mixing associated with the vortex pair in the flow. This factor has been introduced in the diffusivity definition, without any adverse consequences to the mathematical rigour of the model. Finally, it was found that the new entrainment assumption enables the simulation of situations with strong cross-flows, unlike what happens with SE, because the cause of numerical instability was identified and suppressed. Except for the velocity excess of jets in co-flowing streams, where measurement errors are likely to have affected the data, the predictions using the FOC are generally slightly better than Schatzmann's, showing that this closure is a valid alternative. On the other hand, as the new entrainment assumption depends strongly upon the definition of $K$, the results obtained can still be improved if a better way of relating this diffusivity to the mean variables of the equation set is found.

\section{Acknowledgements}

The authors acknowledge the support given by Professor José Pinto Peixoto at the Instituto Geofísico do Infante D. Luís, and by Professor Mendes Victor at the Centro de Geofísica da Universidade de Lisboa, where all the calculations were performed. The authors also thank the useful suggestions given by three anonymous referees. This work has been accomplished with the financial support of JNICT under the Grant PBIC/CTA/2122/95. 


\section{References}

Briggs, G.A.: 1984, 'Plume Rise and Buoyancy Effects',Atmospheric Science and Power Production, D. Randerson, Ed., U.S. Dept. of Energy DOE/TIC-27601, 327-366.

Chu, V.H. and Goldberg, M.B.: 1974, 'Buoyant Forced-plumes in Cross-flow', J. Hydraulics Division of the ASCE 100, 10805-10808.

Csanady, G.T.: 1973, Turbulent Diffusion in the Environment, Geophysics and Astrophysics Monographs, D. Reidel Publishing Company, 248 pp.

Davidson, G.A.: 1986, 'A Discussion of Schatzmann's Integral Plume Model from a Control Volume Viewpoint', J. Climate Appl. Meteorol. 25, 858-867.

Fan, L.N.: 1967, 'Turbulent Buoyant Jets into Stratified or Flowing Ambient Fluids', California Institute of Technology, Report No. KH-R-15, Pasadena.

Fink, L.: 1974, 'Der axialsymmetrische Strahl in einer turbulenten Grundströmung', SFB 80, Universität Karlsruhe, Report No. SFB 80/ET/20

Fox, D.G.: 1970, 'Forced Plume in a Stratified Fluid', J. Geophys. Res. 75, 68186835 .

Hirst, E.: 1971, 'Buoyant Jets Discharged to Quiescent Stratified Ambients', J. Geophys. Res. 76, 7375-7384.

Hirst, E.: 1972, 'Buoyant Jets with Tree-dimensional Trajectories', J. Hydraulics Division of the ASCE 98, 1999-2014.

Hoult, D.P., Fay, J.A. and Forney, L.J.: 1969, 'A theory of plume rise compared with field observations', J. Air Pollut. Control Ass. 19, 585-590.

Middleton, J.H.: 1986, 'The Rise of Forced Plumes in a Stably Stratified Crossflow', Boundary-Layer Meteorol. 36, 137-199.

Morton, B.R.: 1959, 'Forced Plumes', J. Fluid Mech. 5, 151-163.

Morton, B.R., Taylor, G.I. and Turner, J.S., 1956, 'Turbulent Gravitational Convection from Maintained and Instantaneous Sources', Proc. Roy. Soc. London A234, 1-23.

Moussa, Z.M., Trischka, J.W. and Eskinazi, S.: 1977, 'The Near Field in the Mixing of a Round Jet with Cross-stream', J. Fluid Mech. 80, 49-80.

Priestley, C.H.D.: 1956, 'A working theory of the bent-over plume of hot gas', Quart. J. Roy. Met. Soc. 82, 165-176.

Priestley, C.H.D. and Ball, F.K.: 1955, 'Continuous convection from an isolated source of heat', Quart. J. Roy. Meteorol. Soc., 81, 144-157.

Schatzmann, M.: 1978, 'The Integral Equations for Round Buoyant Jets in Stratified Flows', J. Appl. Math. Phys. (ZAMP) 29, 608-630.

Schatzmann, M.: 1979, 'An Integral Model of Plume Rise', Atmos. Environ. 13, 721731.

Schatzmann, M. and Policastro, A.J.: 1984, 'Effects of the Boussinesq Approximation on the Results of Strongly Buoyant Plume Calculations', J. Climate Appl. Meteorol. 23, $117-123$.

Slawson, P.R. and Csanady, G.T.: 1967, 'On the Mean Path of Buoyant, Bent-over Chimney Plumes', J. Fluid Mech. 28, 311-322.

Slawson, P.R. and Csanady, G.T.: 1971, 'The Effect of Atmospheric Conditions on Plume Rise', J. Fluid Mech. 47, 39-49.

Weil, J.C.: 1988, 'Plume Rise', Lectures on Air Pollution Modeling, ed. Akula Venkatram and John C. Wyngaard, 390pp. 


\section{FIGURE CAPTIONS}

Figure 1. The plume coordinate-system

Figure 2. Radius growth for a momentum jet in a co-flowing stream, when $A_{3}=10$ and $A_{3}=2$, using Schatzmann's entrainment formula.

Figure 3. Trajectories of buoyant jets discharged into a homogeneous cross-flow, for the new entrainment definition, with and without taking into account the vortex pair (v. p.).

Figure 4. Dense plumes discharged horizontally into a uniform stagnant ambient fluid.

Figure 5. Buoyant jets discharged at various angles into a stable stratified ambient fluid.

Figure 6. Momentum jets discharged into a co-flowing stream.

Figure 7. Trajectories for momentum jets discharged into homogeneous cross-flows.

Figure 8. Trajectories and centreline concentration decay, for buoyant plumes discharged into a homogeneous cross-flow.

Figure 9. Trajectories of buoyant chimney plumes discharged into stratified cross-winds.

Figure 10. Same as Figure 6, but with the entrainment definition given by (53)

Figure 11. Trajectory and radius of a weak plume in a cross-flow, for Schatzmann's entrainment definition and for the FOC. Conditions at the end of the flow establishment zone are: $\mathrm{F}{ }_{r}=10, U_{a} / u_{0}^{*}=1, \theta_{0}=30^{\circ}, \Theta_{0}^{*} / \Theta_{a}=0.1$. 


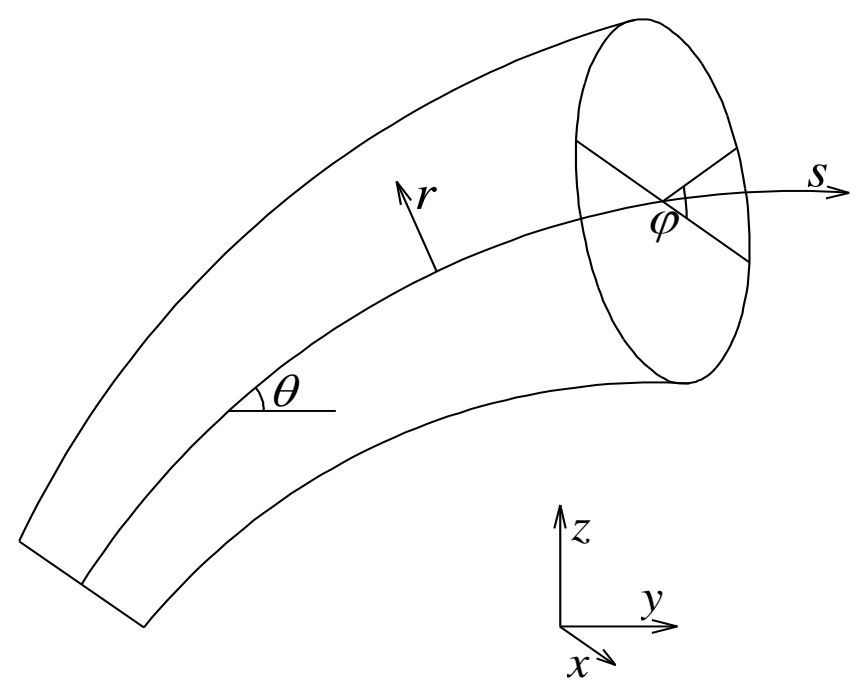

Figure 1. (Teixeira and Miranda) 


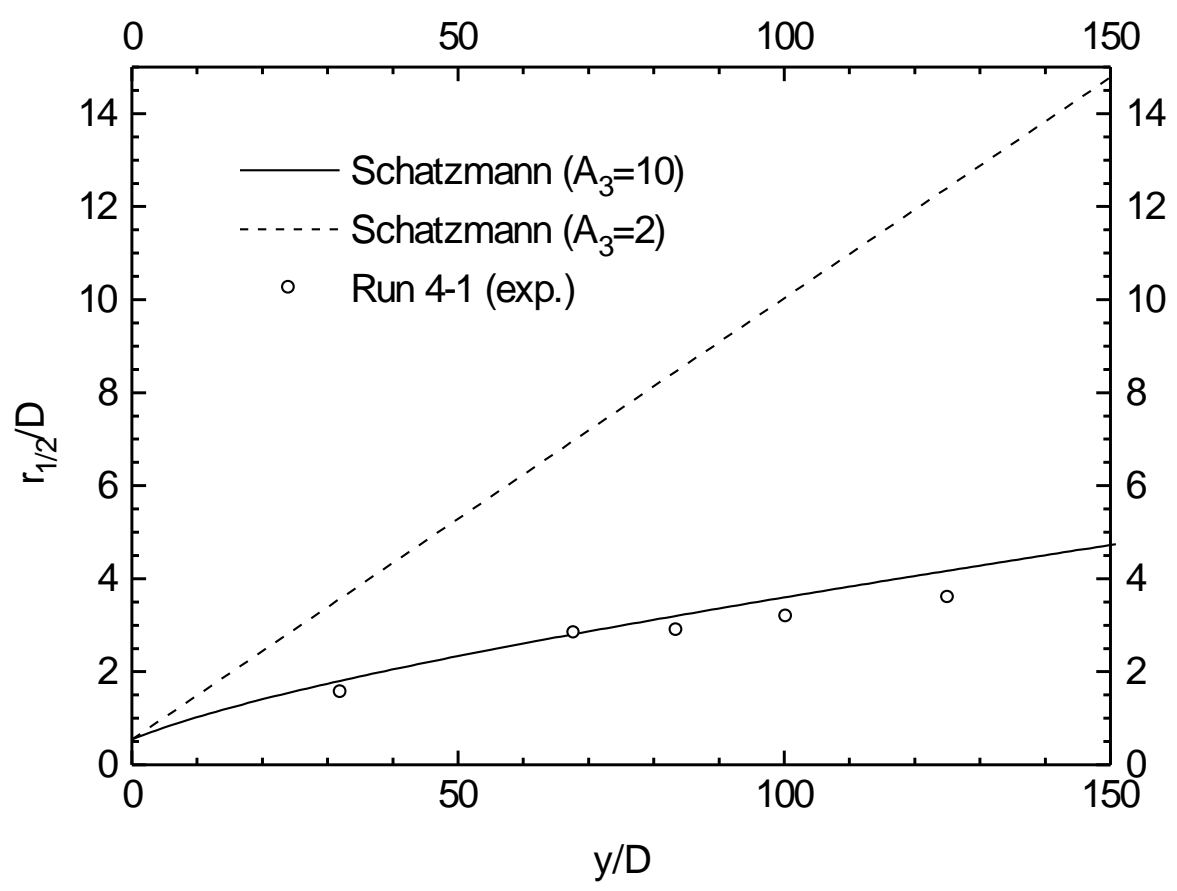

Figure 2. (Teixeira and Miranda) 


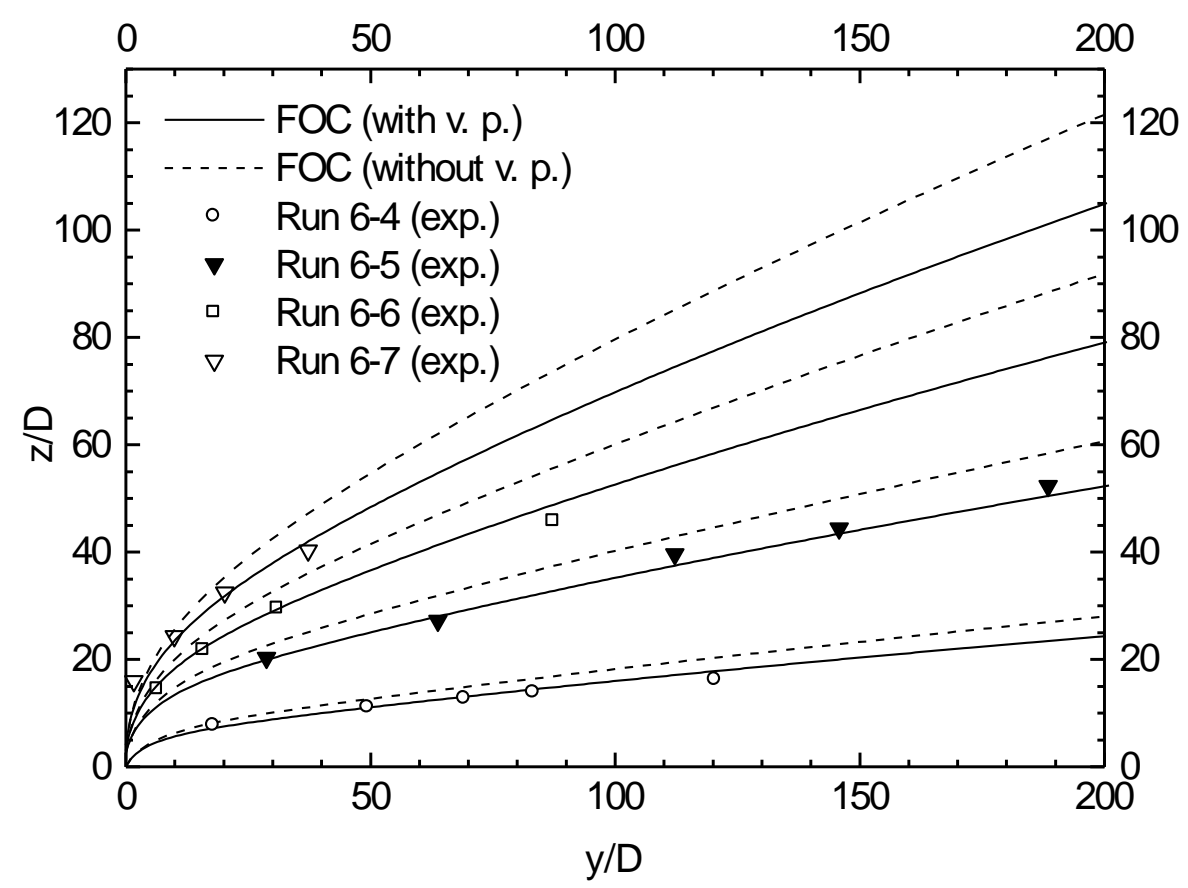

Figure 3. (Teixeira and Miranda) 


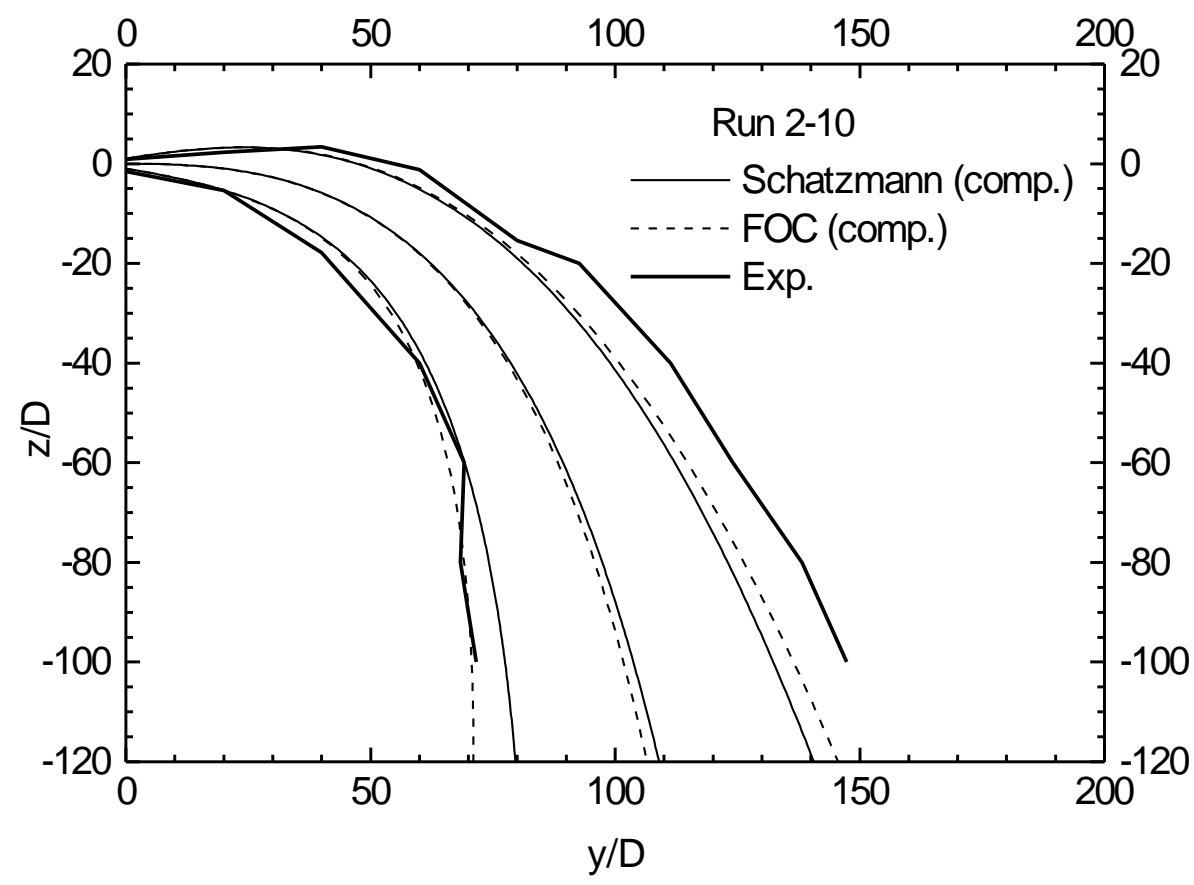

Figure 4a. (Teixeira and Miranda) 


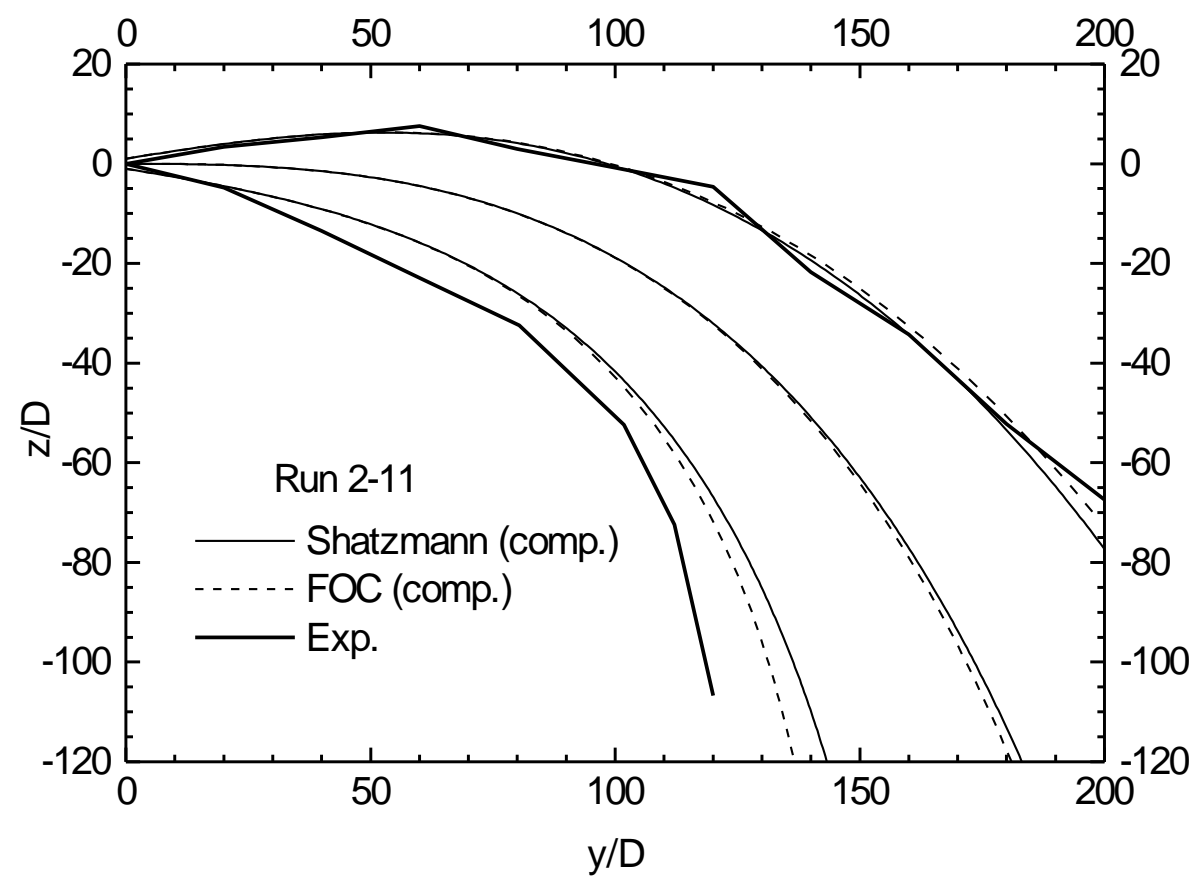

Figure 4b. (Teixeira and Miranda) 


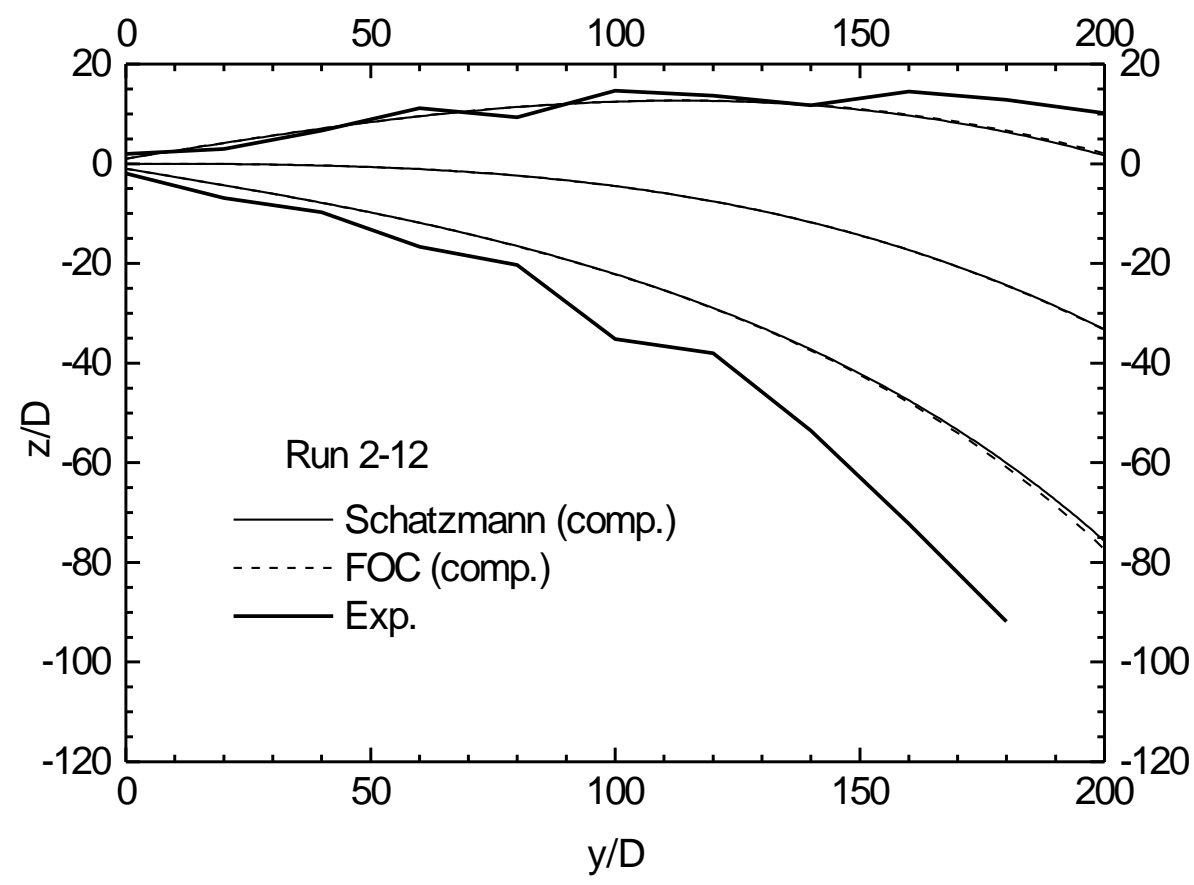

Figure 4c. (Teixeira and Miranda) 


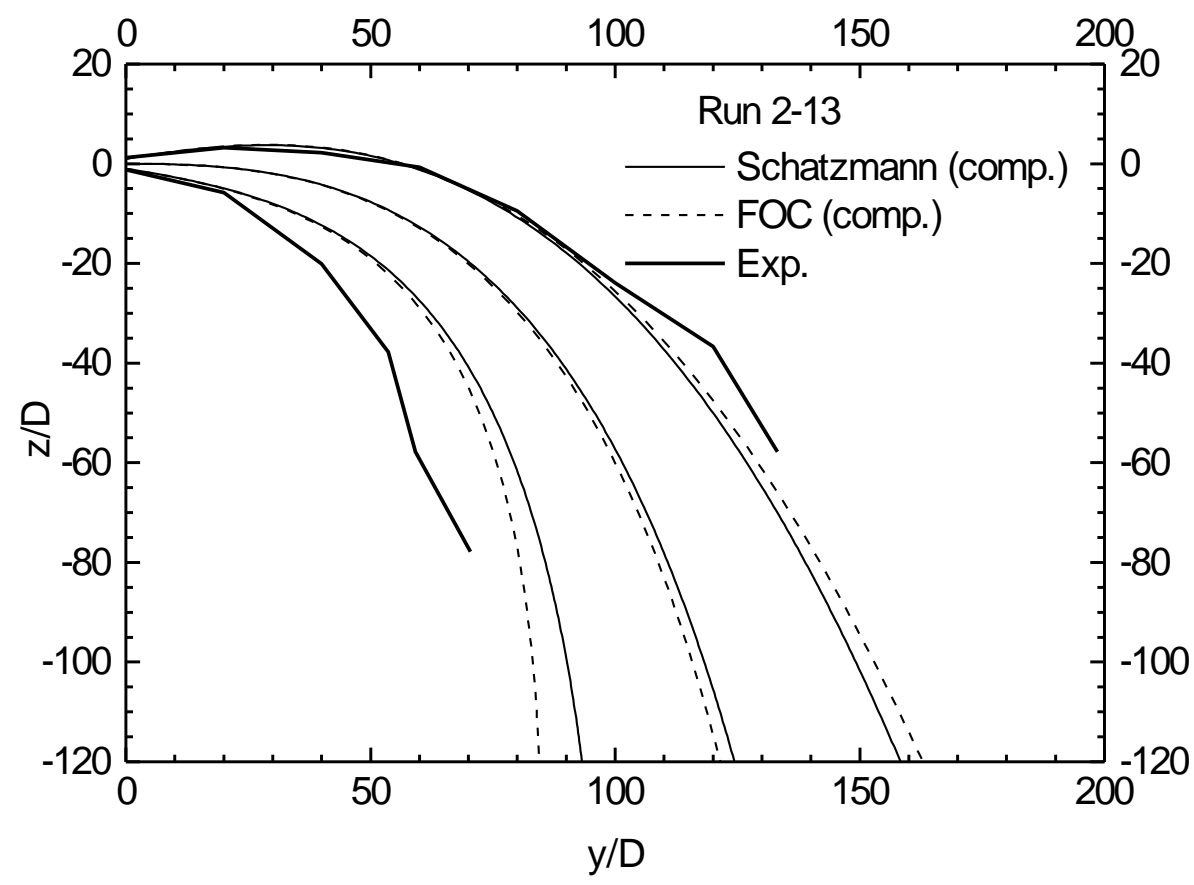

Figure 4d. (Teixeira and Miranda) 


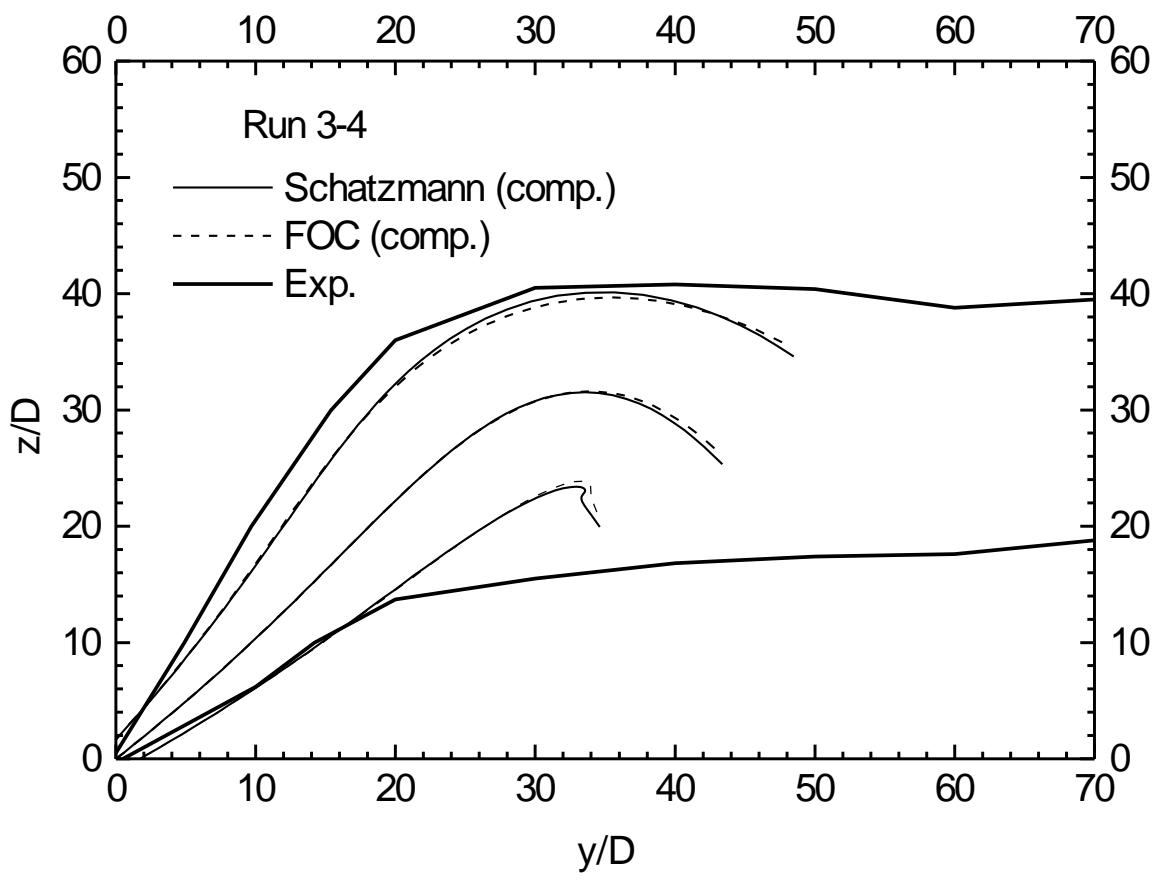

Figure 5a. (Teixeira and Miranda) 


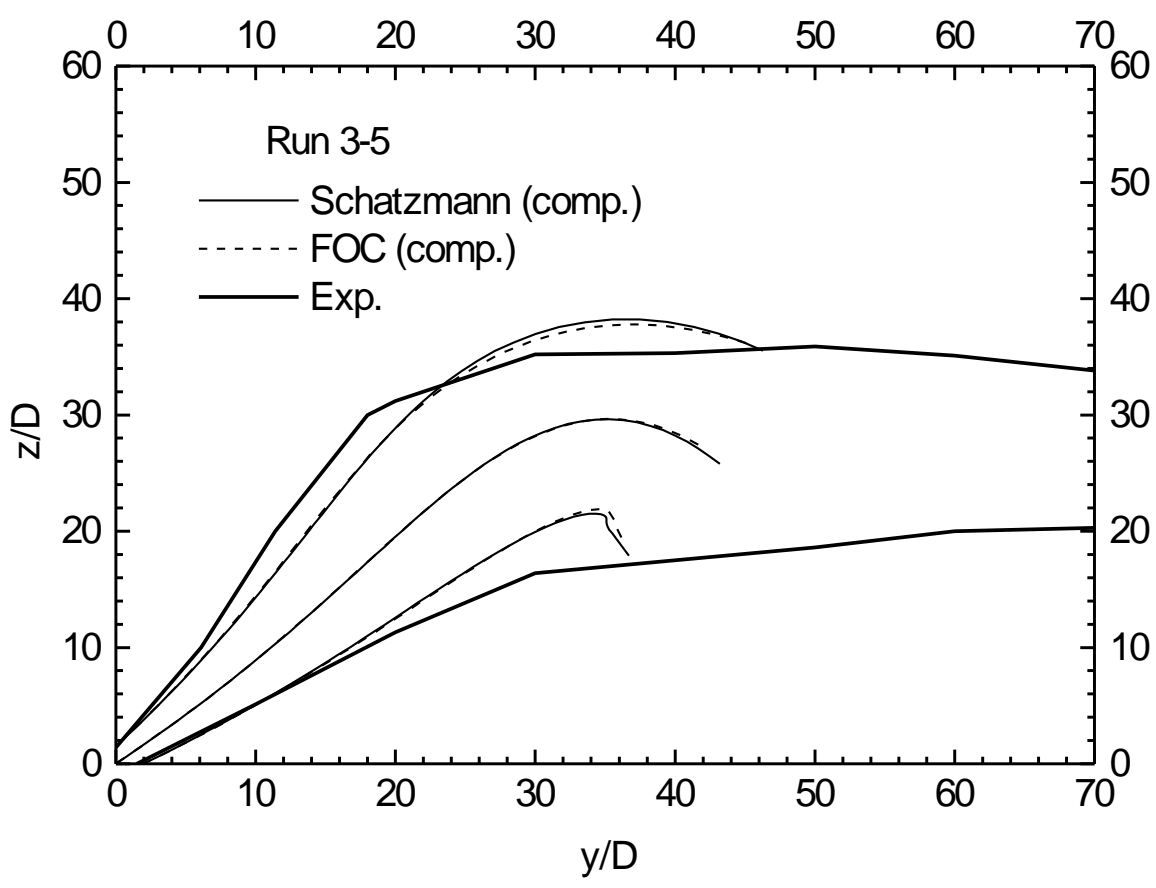

Figure 5b. (Teixeira and Miranda) 


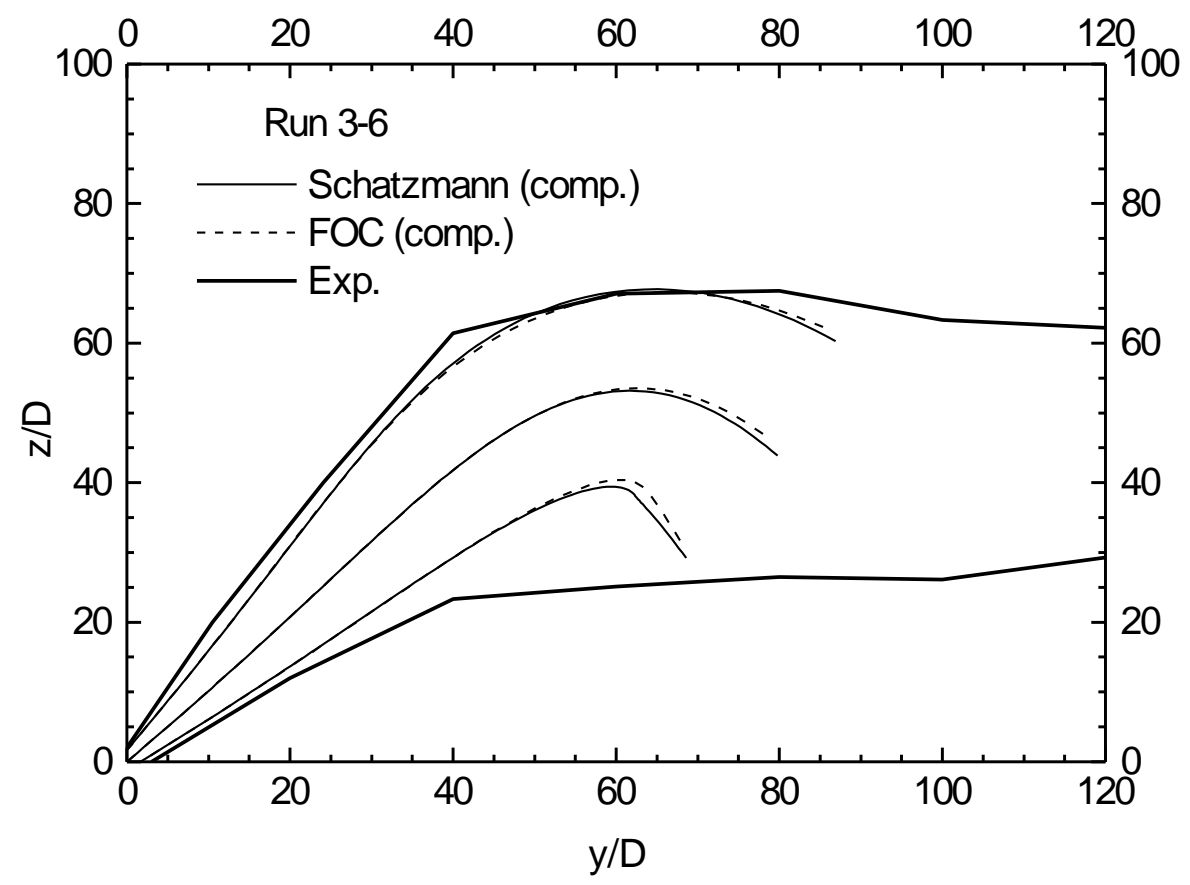

Figure 5c. (Teixeira and Miranda) 


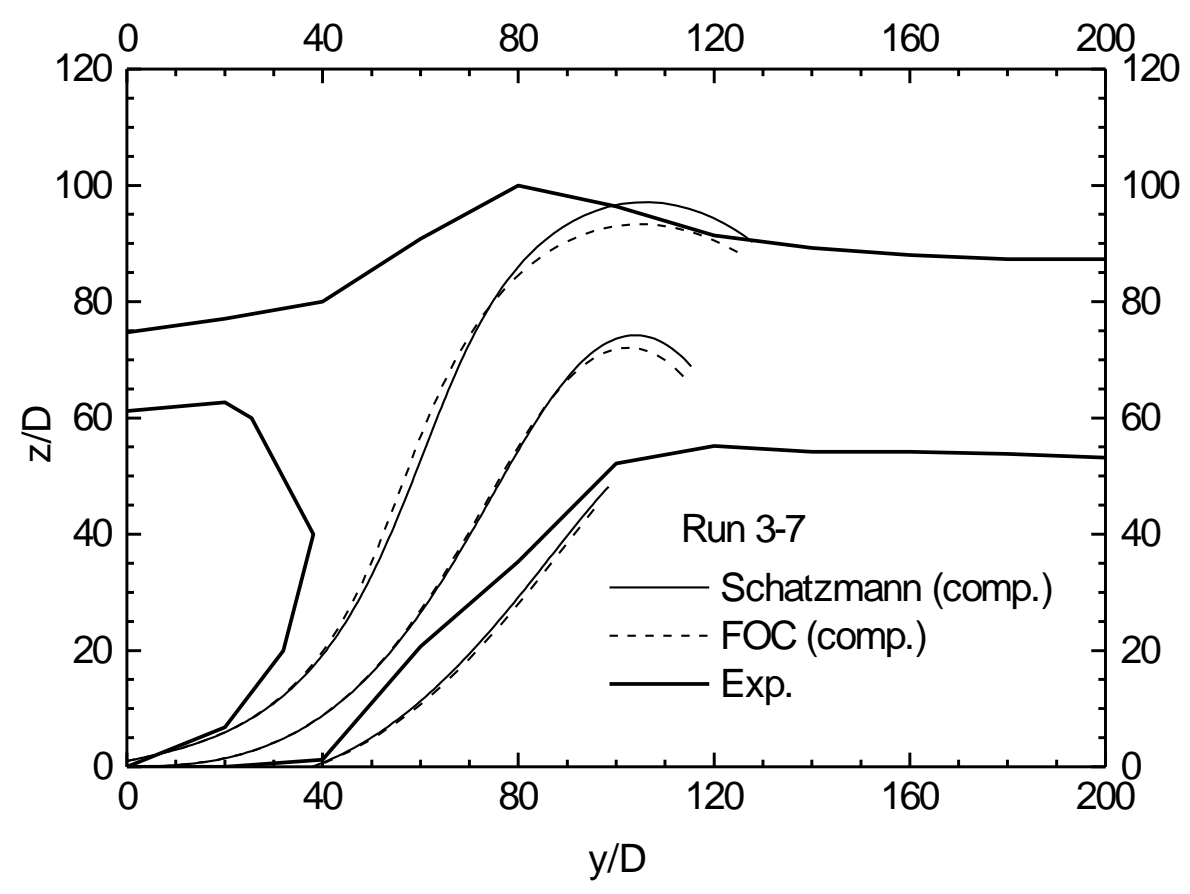

Figure 5d. (Teixeira and Miranda) 


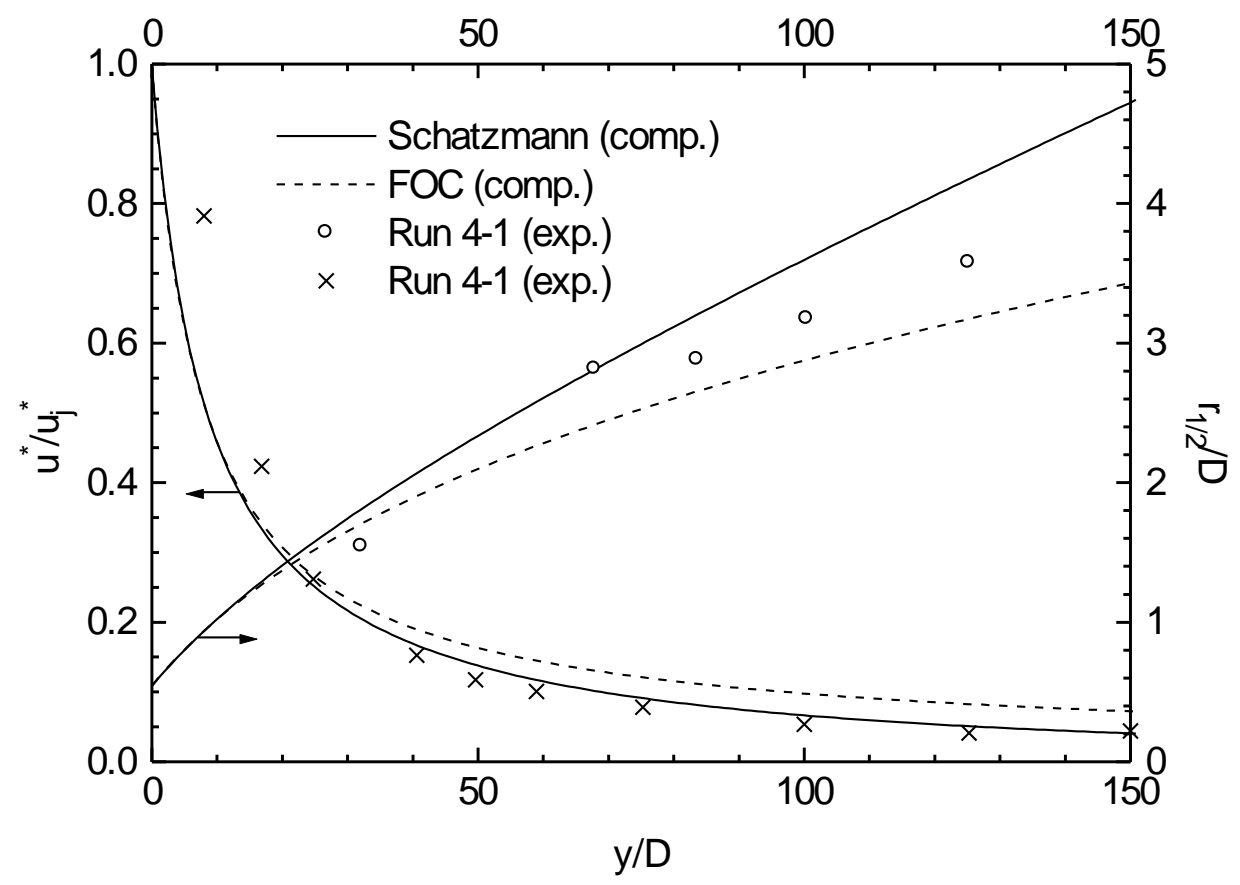

Figure 6a. (Teixeira and Miranda) 


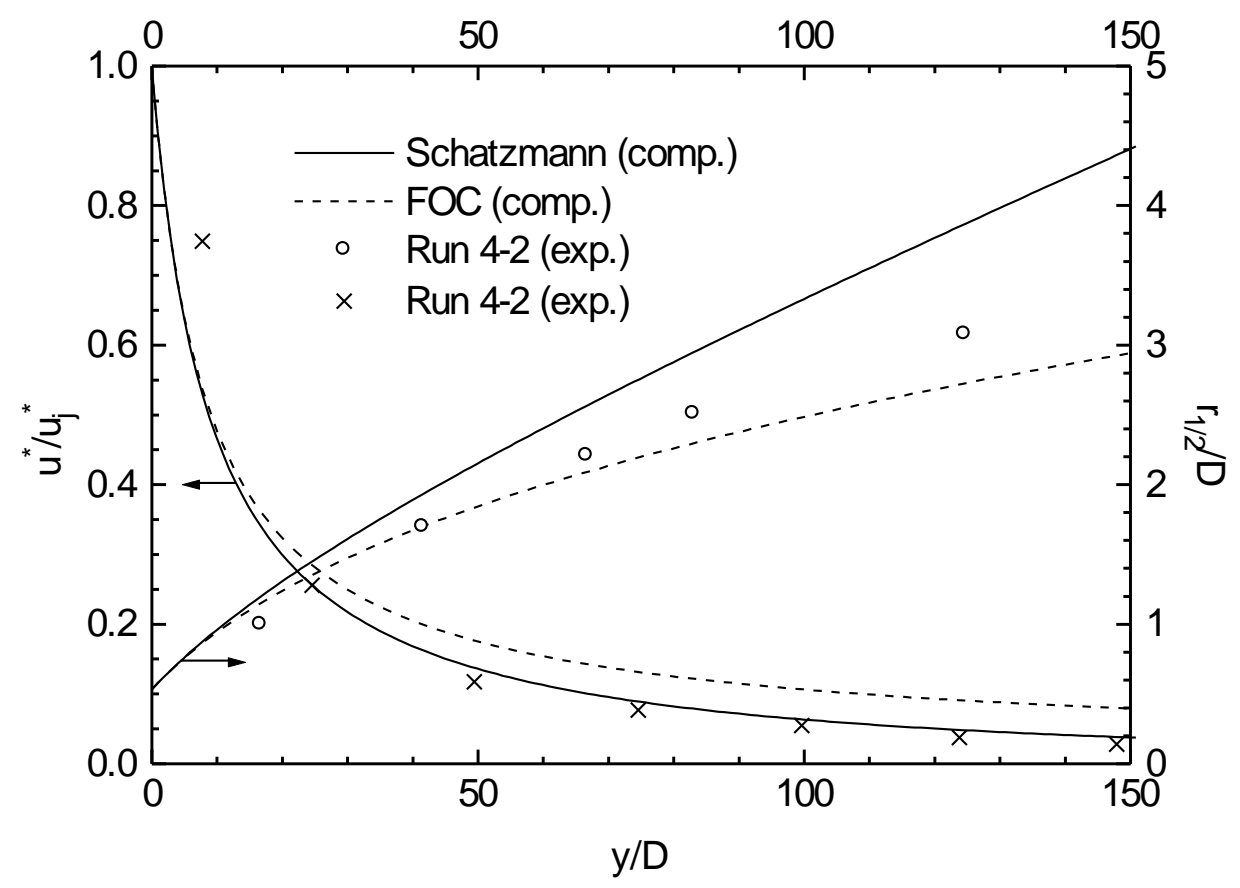

Figure 6b. (Teixeira and Miranda) 


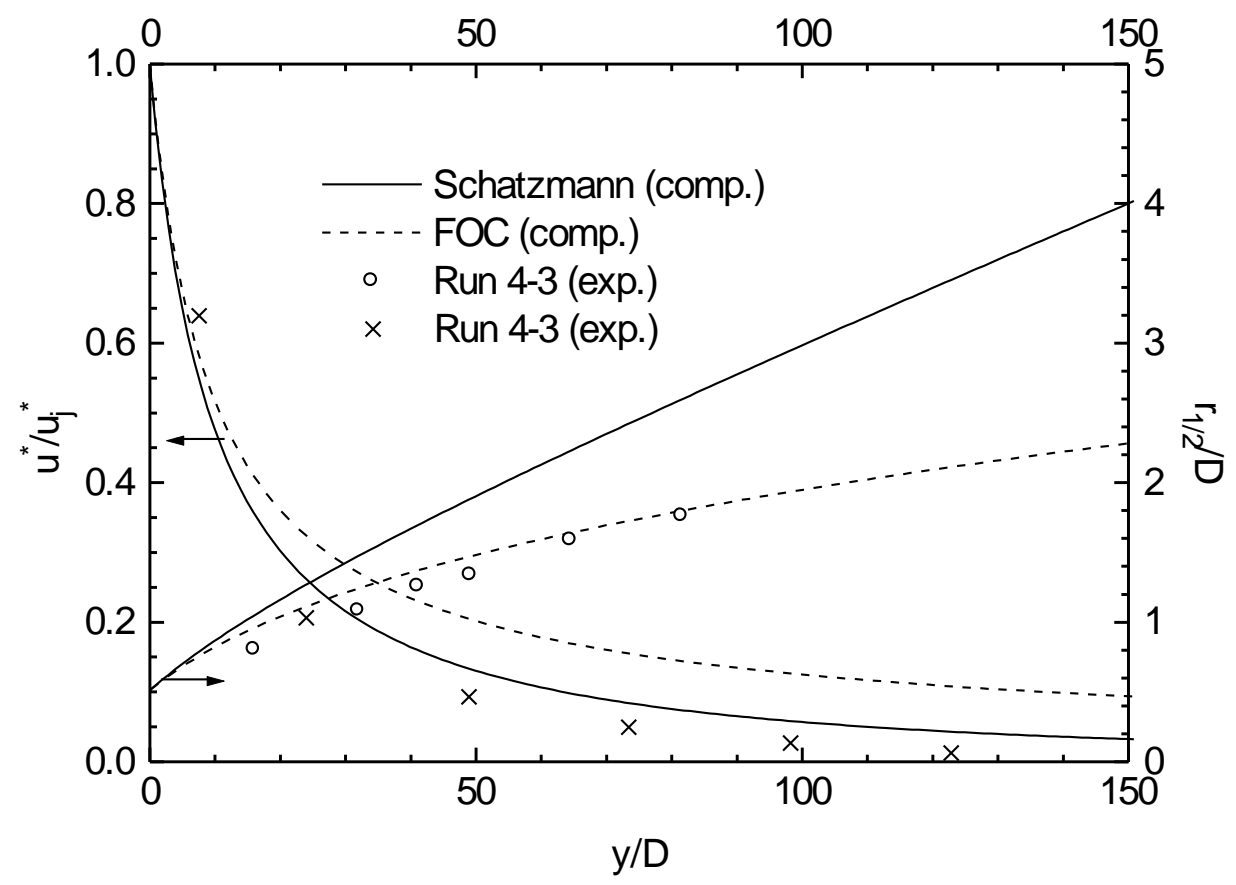

Figure 6c. (Teixeira and Miranda) 


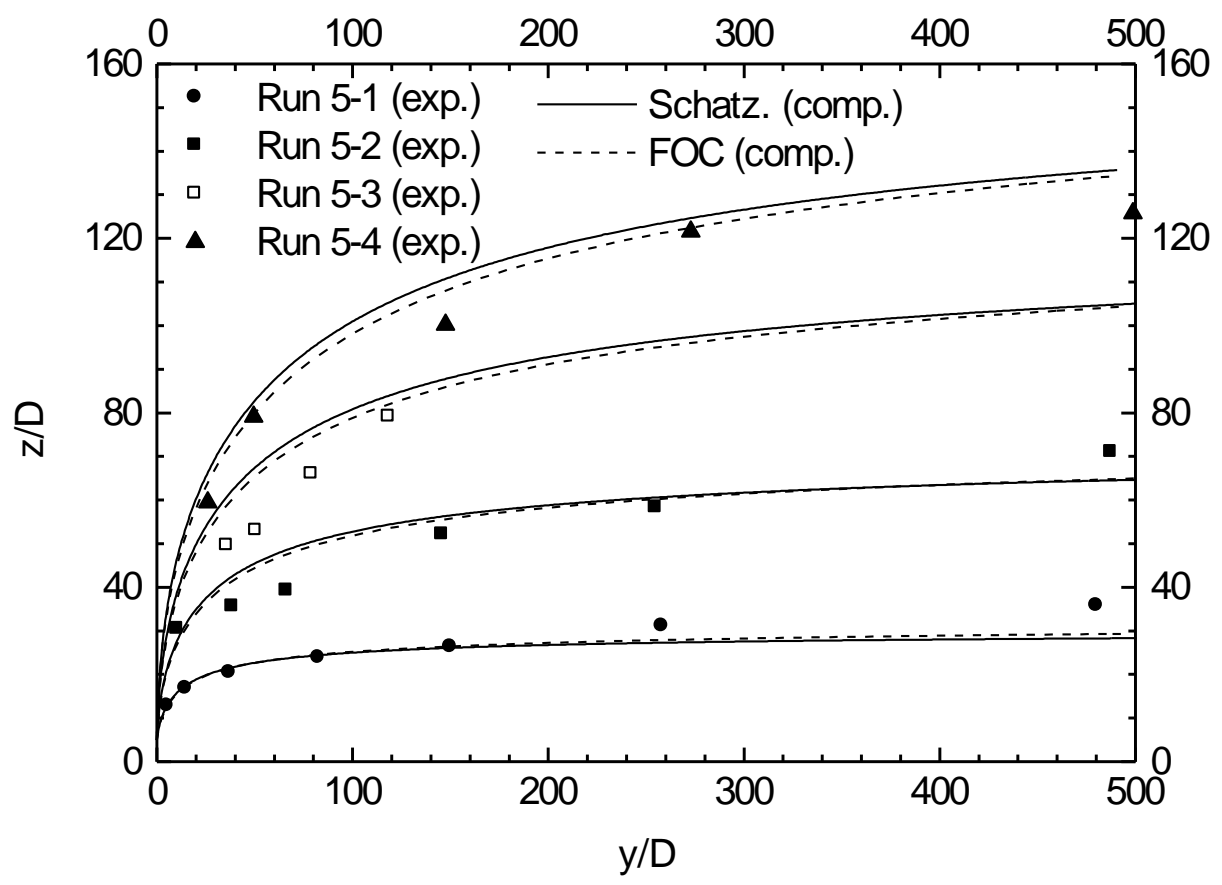

Figure 7. (Teixeira and Miranda) 


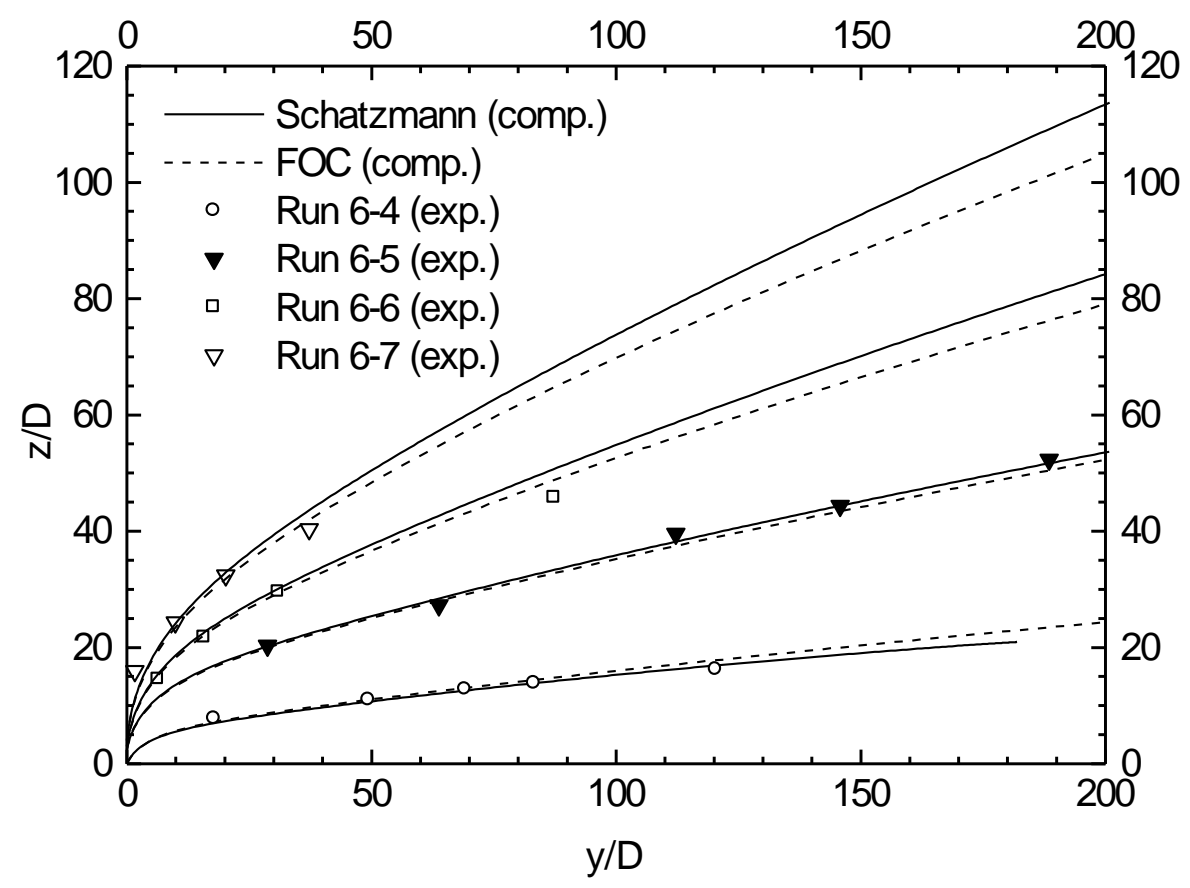

Figure 8a. (Teixeira and Miranda) 


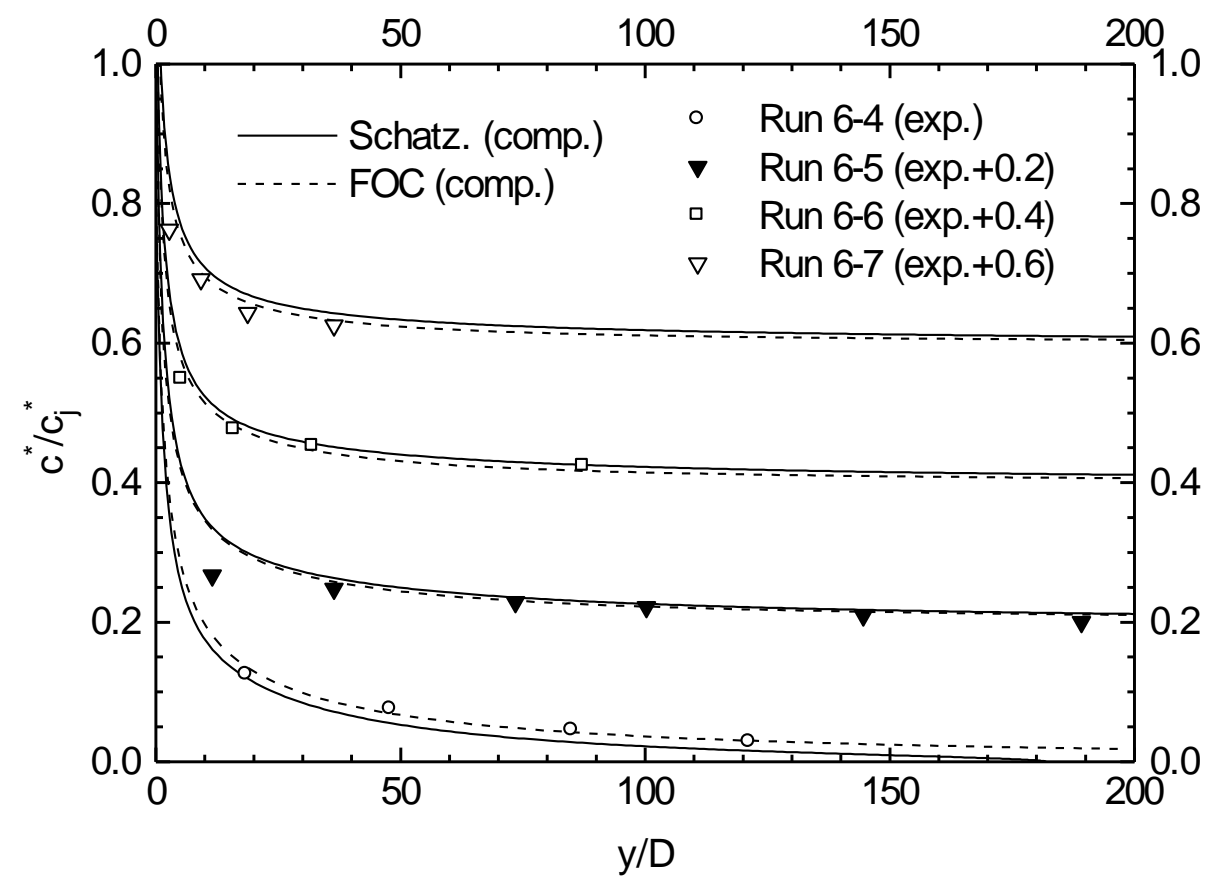

Figure 8b. (Teixeira and Miranda) 


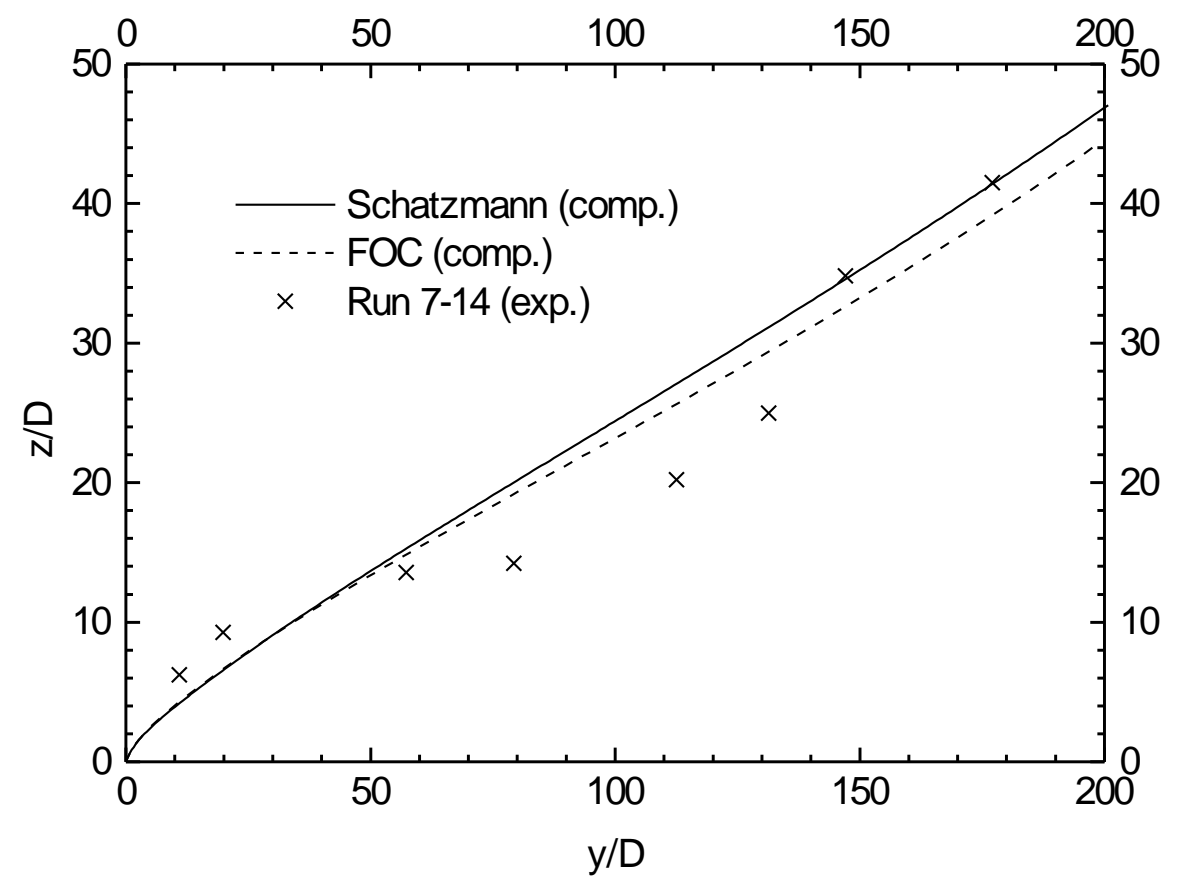

Figure 9a. (Teixeira and Miranda) 


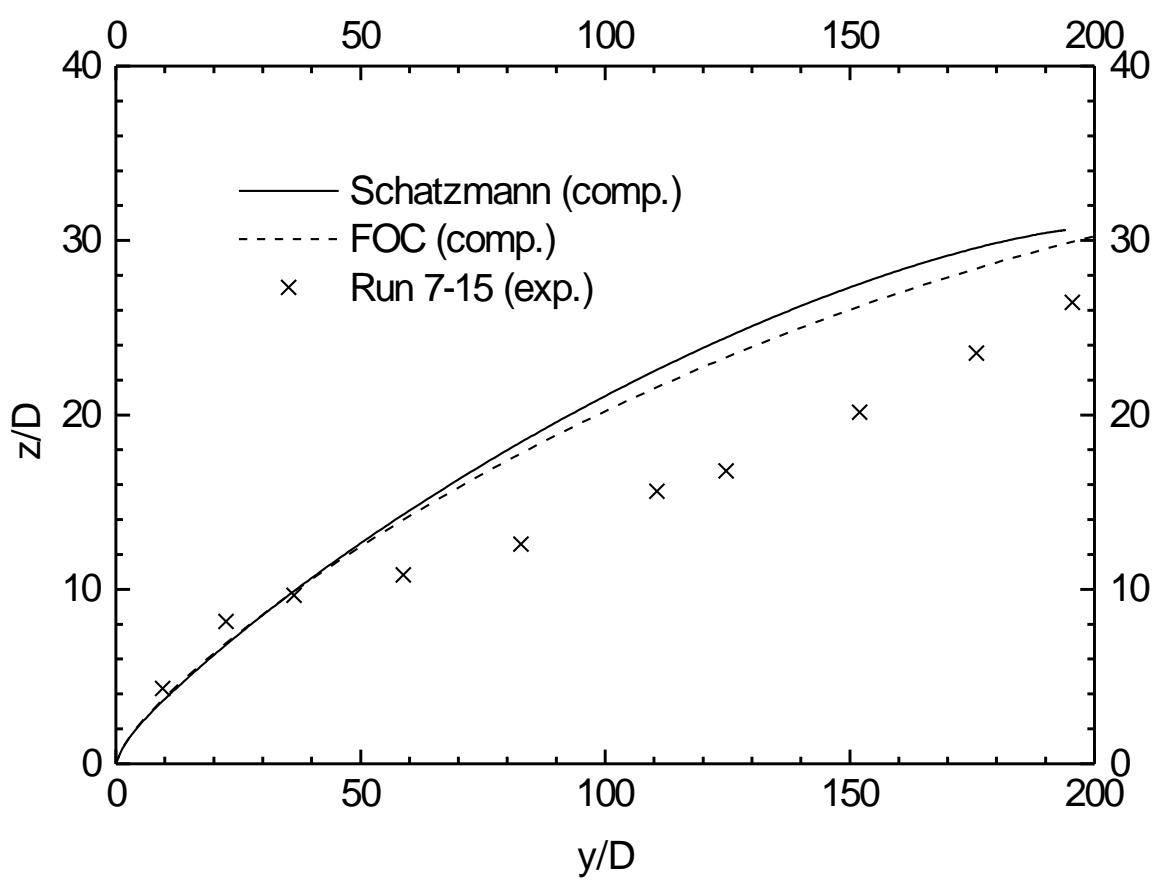

Figure 9b. (Teixeira and Miranda) 


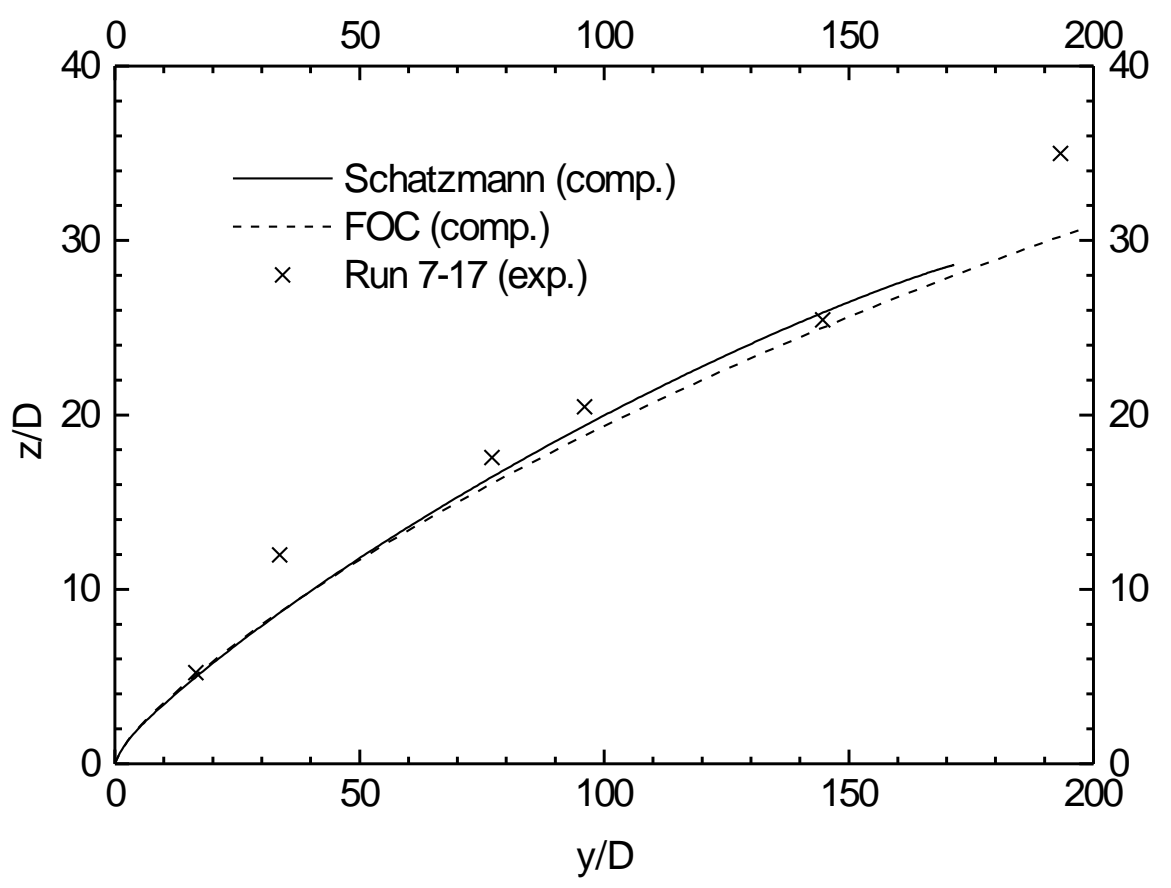

Figure 9c. (Teixeira and Miranda) 


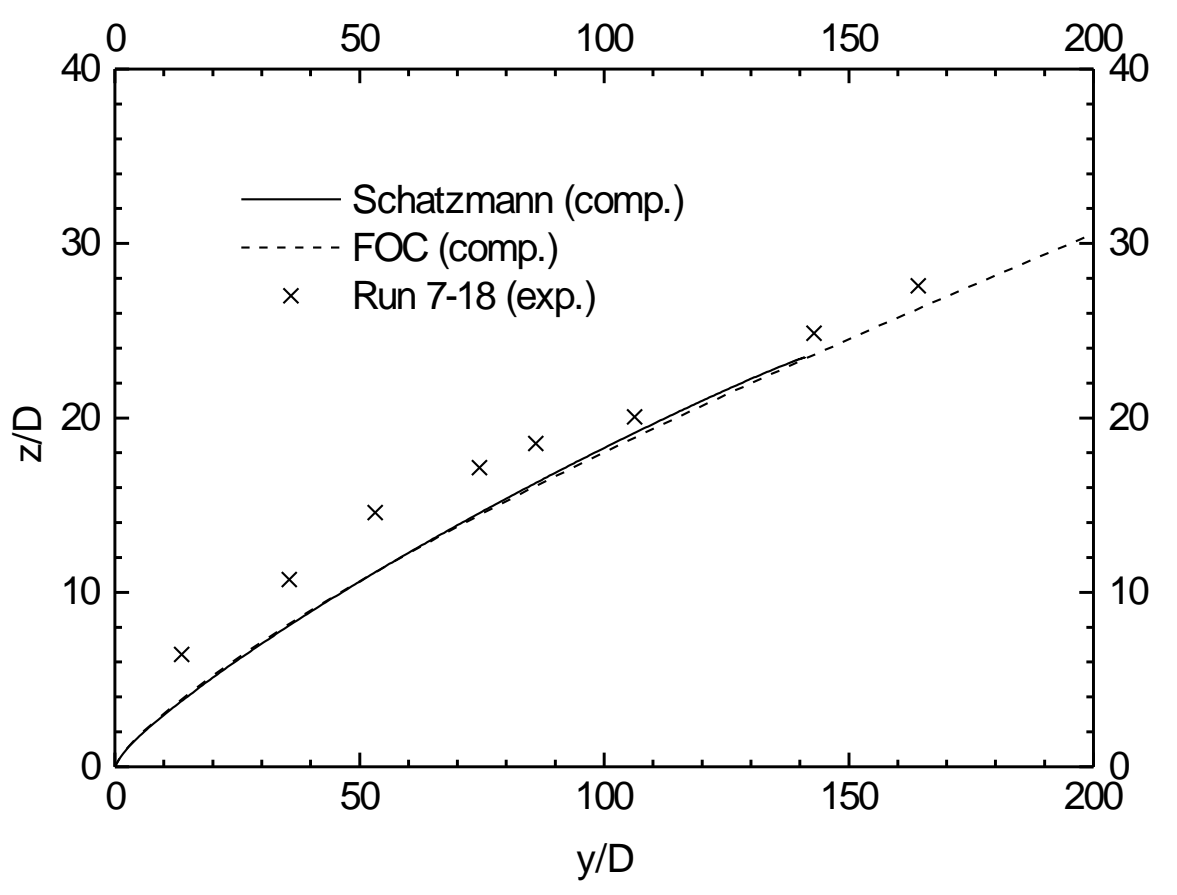

Figure 9d. (Teixeira and Miranda) 


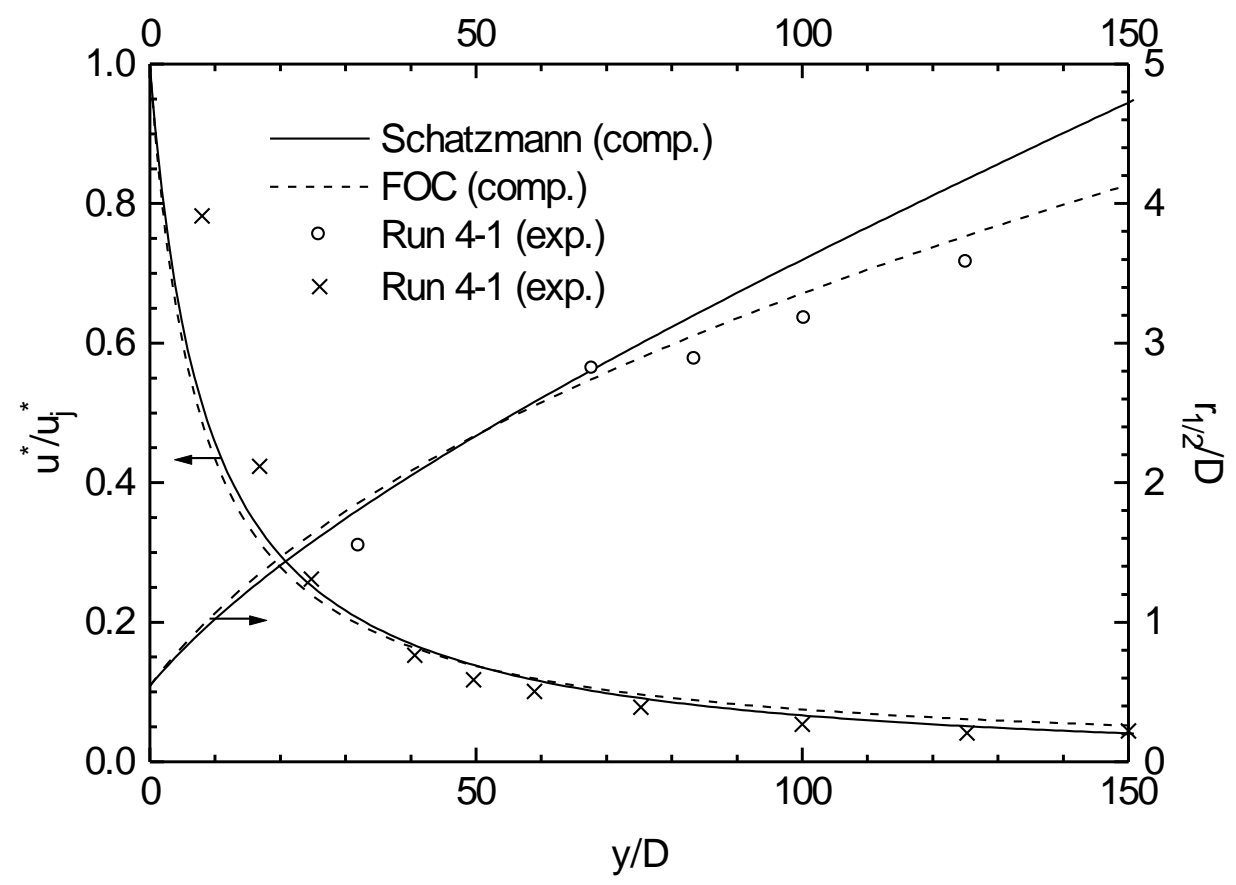

Figure 10a. (Teixeira and Miranda) 


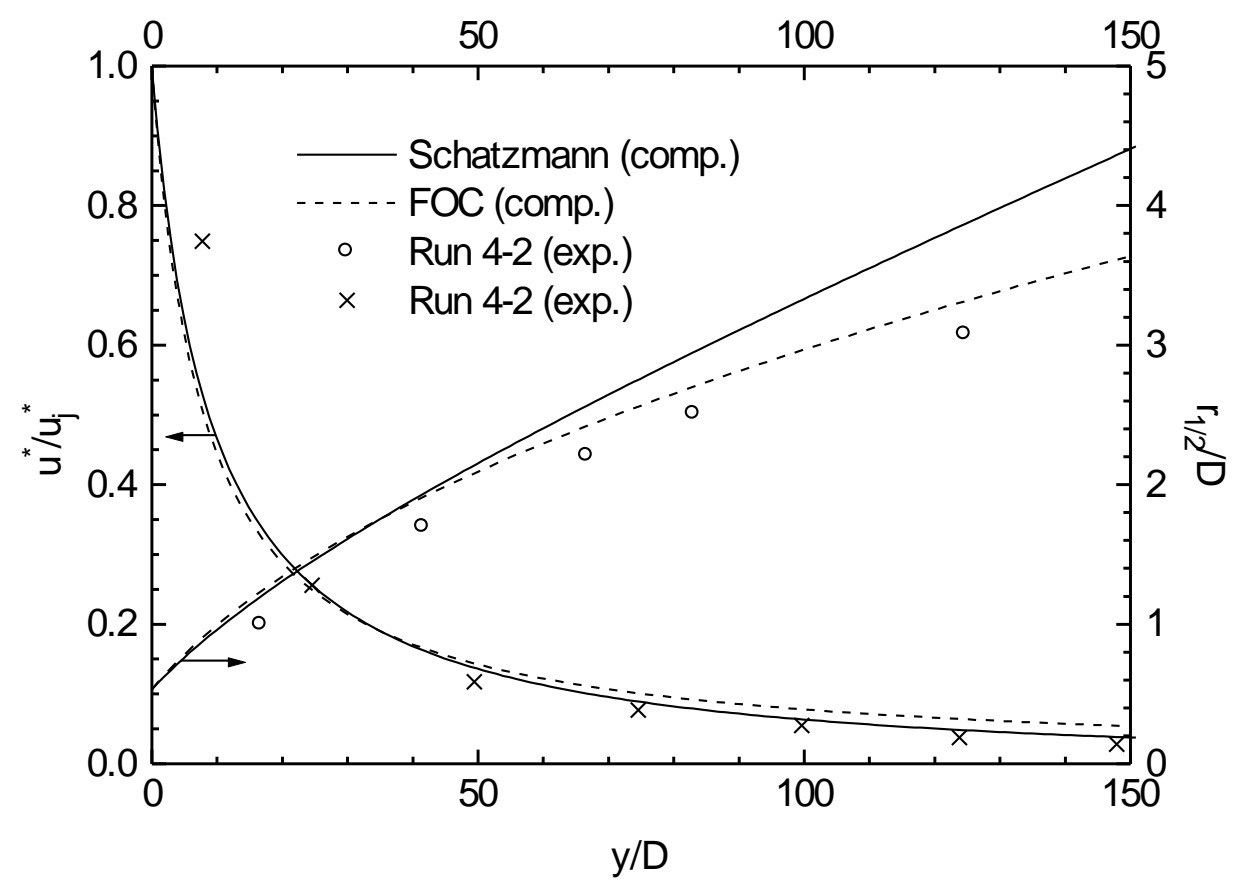

Figure 10b. (Teixeira and Miranda) 


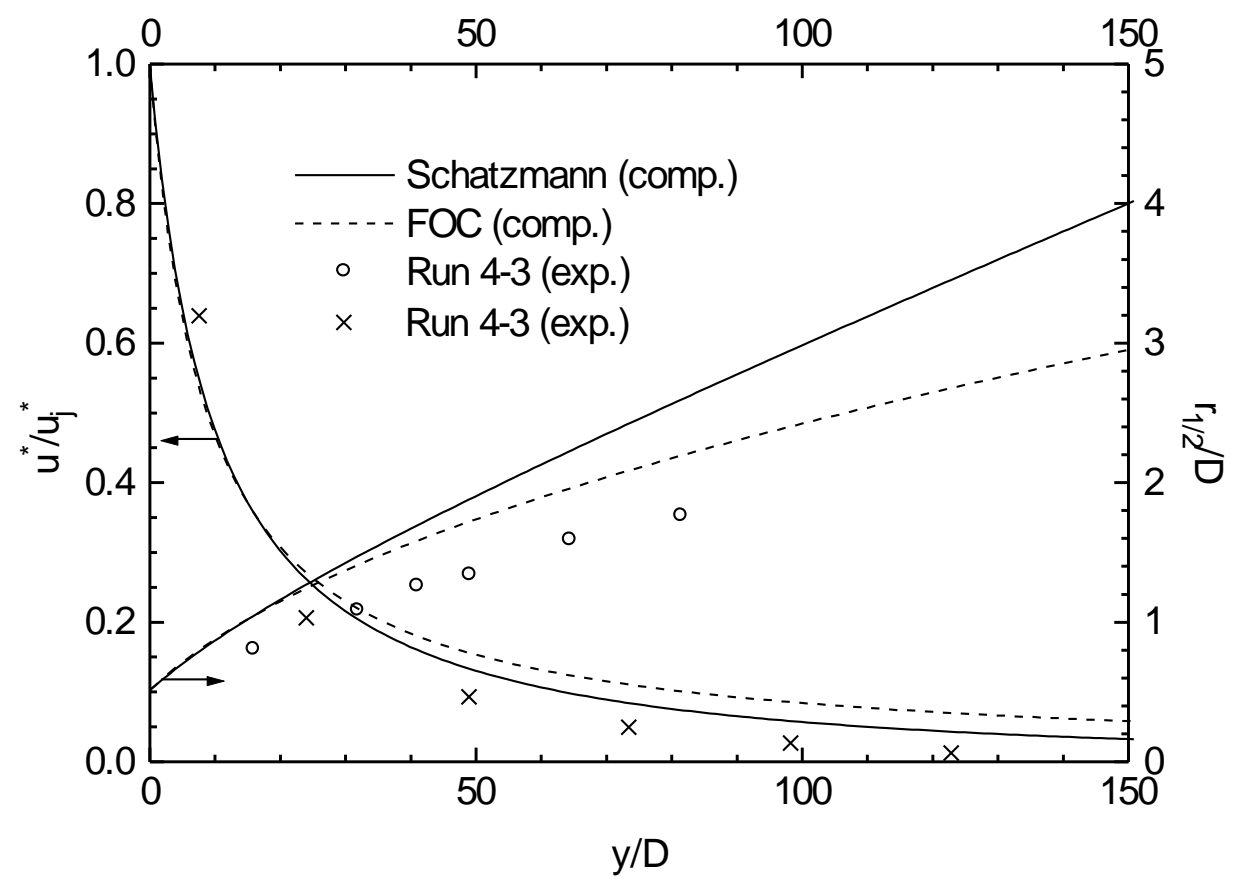

Figure 10c. (Teixeira and Miranda) 


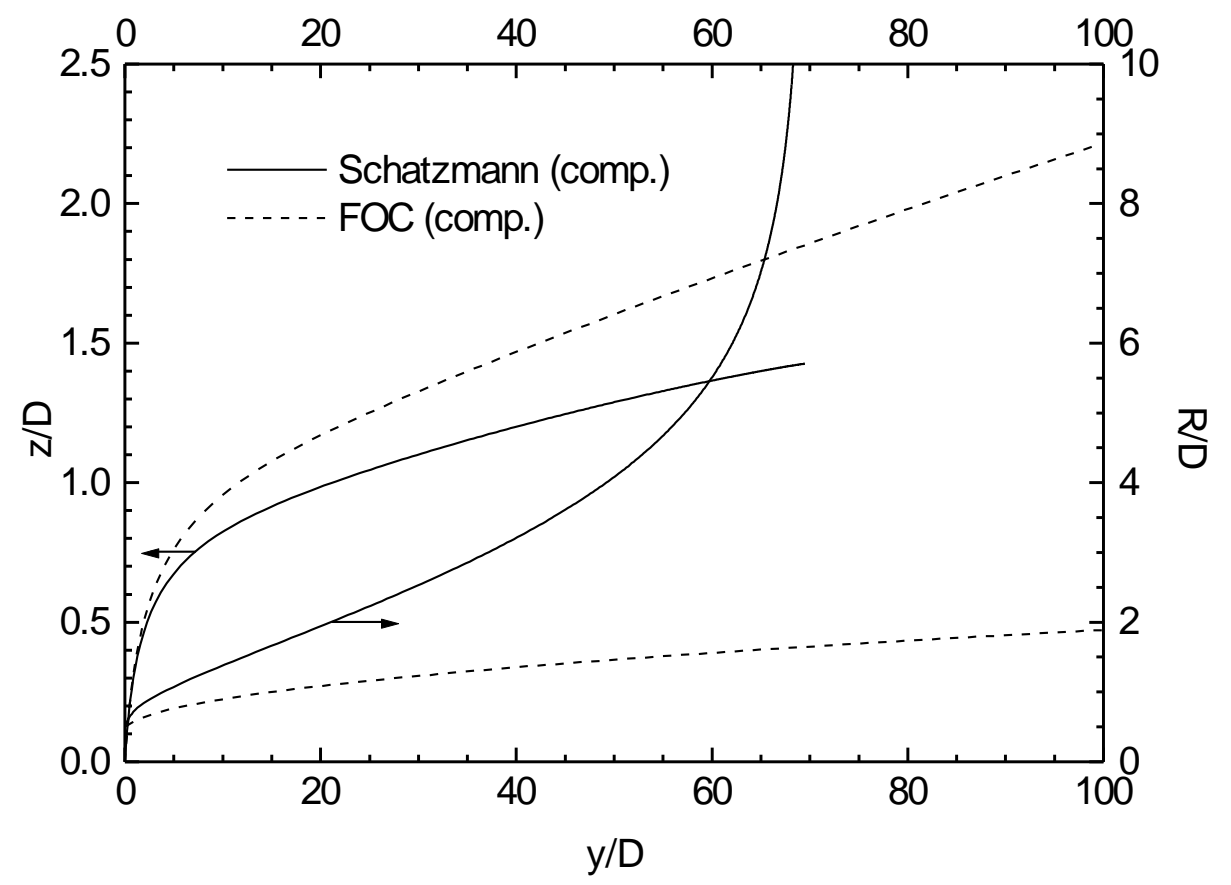

Figure 11. (Teixeira and Miranda) 